\title{
Quantum caustics and the hierarchy of light cones in quenched spin chains
}

\author{
W. Kirkby $\odot,{ }^{1}$ J. Mumford $\odot,{ }^{1,2}$ and D. H. J. O’Dell $\odot^{1, *}$ \\ ${ }^{1}$ Department of Physics and Astronomy, McMaster University, 1280 Main St. W., Hamilton, Ontario, Canada L8S $4 M 1$ \\ ${ }^{2}$ School of Arts and Sciences, Red Deer College, 100 College Blvd., Red Deer, Alberta, Canada T4N 5 H5
}

(Received 14 November 2018; revised manuscript received 25 October 2019; published 27 November 2019)

\begin{abstract}
We show that the light conelike structures that form in spin chains after a quench are quantum caustics. Their natural description is in terms of catastrophe theory and this implies (1) a hierarchy of light cone structures corresponding to the different catastrophes, (2) dressing by characteristic wave functions that obey scaling laws determined by the Arnol'd and Berry indices, and (3) a network of vortex-antivortex pairs in space-time inside the cone. We illustrate the theory by giving explicit calculations for the transverse field Ising model and the XY model, finding fold catastrophes dressed by the Airy functions and cusp catastrophes dressed by the Pearcey functions; multisite correlation functions are described by higher catastrophes such as the hyperbolic umbilic. Furthermore, we find that the vortex pairs created inside the cone are sensitive to phase transitions in these spin models with their rate of production being determined by the dynamical critical exponent. More broadly, this work illustrates how catastrophe theory can be applied to singularities in quantum fields.
\end{abstract}

DOI: 10.1103/PhysRevResearch.1.033135

\section{INTRODUCTION}

According to Lieb and Robinson [1], there is a maximum speed $v_{\mathrm{LR}}$ at which information can propagate in discrete quantum systems that obey the Schrödinger equation and have short range interactions. This is a powerful and generic statement because it implies that, despite the fact there is no intrinsic speed limit in the (nonrelativisitic) Schrödinger equation, the response of these many-particle systems to a sudden quench should be in terms of a light conelike time evolution of spatial correlations [2]. Physically, the "light cone" arises from the maximum group velocity of quasiparticles that are excited by the quench and that subsequently propagate through the sample [3]. Sophisticated methods of analysis have been applied to these quench problems including conformal field theory and tensor networks [3-16], and the theory has been tested in experiments on ultracold atoms [17-19] and ions [20,21] where quantum spin models [18,22-25], the Bose-Hubbard (BH) model [26-29], 1D systems [30-32], and quantum walks on a lattice $[33,34]$ can all be realized. The long coherence times of atomic systems make them particularly suited to studying such dynamics [35,36], and the ability to perform single-site manipulation and detection [37-40] has enabled unprecedented preparation and visualization of the relevant local observables.

In this paper, we show that light cones in quenched spin chains are quantum caustics. These are quantum versions of wave focusing phenomena that occur widely in nature in the form of rainbows [41], ship wakes [42-44], tsunamis and tidal

\footnotetext{
*Corresponding author: dodell@mcmaster.ca

Published by the American Physical Society under the terms of the Creative Commons Attribution 4.0 International license. Further distribution of this work must maintain attribution to the author(s) and the published article's title, journal citation, and DOI.
}

bores [45], and Cherenkov radiation [46] (including superfluid analogs [47-49]). In the geometric ray theory, caustics occur where two or more rays coalesce, giving regions in space where the intensity diverges. By virtue of their singular nature, the natural mathematical description of caustics is via catastrophe theory which partitions them into a hierarchy of equivalence classes, each of which is structurally stable and has its own set of scaling relations [50-52]. To show specifically how this approach can be applied to spin chains, we consider the exactly solvable $1 \mathrm{D}$ XY model $[53,54]$, as well as the special case of the 1D transverse-field Ising model (TFIM) [55,56]. While both cases display light conelike behavior, the more general XY model allows for an anisotropic coupling giving rise to a double cone [57,58]. Although we limit our calculations to these exactly solvable models, the structural stability of catastrophes (insensitivity to small perturbations) guarantees they must survive in the presence of weak nonintegrability. This includes weak interactions between quasiparticles or disorder and therefore our results also apply to more general systems than just exactly solvable models.

Wave interference softens caustics and leads to structure on three scales [52]: at large scales, we see divergent ray caustics, whereas at wavelength scales interference smoothes the divergences and dresses each caustic with a characteristic wave function which in the simplest case of two coalescing rays is the Airy function, and finally at subwavelength scales there are networks of vortex-antivortex pairs. These robust features, including vortex-antivortex networks, have been observed in optical fields [41], and more recently in electron microscopy [69]. They have also been discussed theoretically in the context of Bose-Einstein condensates $[73,74]$ and various aspects seen experimentally in these systems [70-72]. Furthermore, the association between the Airy function (and its related kernels) and light cones has previously been noted by various authors $[8,14,15,59-64]$, and recent work has conjectured similar universal forms for wavefronts of out-of-timeordered correlators [65-68] by examining asymptotic limits of 
TABLE I. The seven elementary catastrophes and their generating functions $\Phi_{Q}(\mathbf{s} ; \mathbf{C})$, organized by corank $n$, and dimension $Q$ of control space [86]. The associated Arnol'd exponents $\beta_{Q}$ and Berry exponents $\varsigma_{m}$ governing the scaling of the wave catastrophes' amplitudes and phase, respectively, are also listed.

\begin{tabular}{|c|c|c|c|c|c|}
\hline \multirow[b]{2}{*}{ Catastrophe } & \multicolumn{3}{|r|}{ Generating function } & \multicolumn{2}{|r|}{ Scaling exponents } \\
\hline & $n$ & $Q$ & $\Phi_{Q}(\mathbf{s} ; \mathbf{C})$ & $\beta_{Q}$ & $\left\{\varsigma_{m}\right\}$ \\
\hline Fold & 1 & 1 & $s^{3} / 3+C s$ & $1 / 6$ & $\varsigma=2 / 3$ \\
\hline Cusp & 1 & 2 & $s^{4} / 4+C_{2} s^{2} / 2+C_{1} s$ & $1 / 4$ & $\varsigma_{1}=3 / 4, \varsigma_{2}=1 / 2$ \\
\hline Swallowtail & 1 & 3 & $s^{5} / 5+C_{3} s^{3} / 3+C_{2} s^{2} / 2+C_{1} s$ & $3 / 10$ & $\varsigma_{1}=4 / 5, \varsigma_{2}=3 / 5, \varsigma_{3}=2 / 5$ \\
\hline Butterfly & 1 & 4 & $s^{6} / 6+C_{4} s^{4} / 4+C_{3} s^{3} / 3+C_{2} s^{2} / 2+C_{1} s$ & $1 / 3$ & $\varsigma_{1}=5 / 6, \varsigma_{2}=2 / 3, \varsigma_{3}=1 / 2, \varsigma_{4}=1 / 3$ \\
\hline Hyperbolic Umbilic & 2 & 3 & $s_{1}^{3} / 3+s_{2}^{3} / 3+C_{3} s_{1} s_{2}+C_{2} s_{2}+C_{1} s_{1}$ & $1 / 3$ & $\varsigma_{1}=2 / 3, \varsigma_{2}=2 / 3, \varsigma_{3}=1 / 3$ \\
\hline Elliptic Umbilic & 2 & 3 & $3 s_{1}^{2} s_{2}-s_{2}^{3}+C_{3}\left(s_{1}^{2}+s_{2}^{2}\right)+C_{2} s_{2}+C_{1} s_{1}$ & $1 / 3$ & $\varsigma_{1}=2 / 3, \varsigma_{2}=2 / 3, \varsigma_{3}=1 / 3$ \\
\hline Parabolic Umbilic & 2 & 4 & $s_{2}^{4}+s_{1}^{2} s_{2}+C_{4} s_{2}^{2}+C_{3} s_{1}^{2}+C_{2} s_{2}+C_{1} s_{1}$ & $3 / 8$ & $\varsigma_{1}=5 / 8, \varsigma_{2}=3 / 4, \varsigma_{3}=1 / 2, \varsigma_{4}=1 / 4$ \\
\hline
\end{tabular}

the Airy function. However, to the best of our knowledge, the present paper is the first to study the hierarchy of universal wave functions that dress light cones, of which the Airy function is only the first, and also point out that light cones should generically contain networks of vortices which in the case of 1D chains appear as space-time vortices.

A fourth scale appears in quantum fields due to discretization of excitations leading to "quantum catastrophes" [75-80] (rippling mirrors give analogous effects [81]). Going to the continuum (classical field) limit returns us to a wave catastrophe. As we shall show, light cones in spin chains have all the features of quantum catastrophes, including discretized versions of wave catastrophes and vortices which are regulated by the lattice constant. Although the cone itself is mildly affected by the presence of a quantum critical point (QCP) in the spin models we study, we find by contrast that the vortices are strongly affected and we use this feature to extract the dynamical critical scaling.

The rest of this paper is organized as follows. In Sec. II, we outline the relevant aspects of catastrophe theory, emphasizing the hierarchy of structures and their scaling properties. In Sec. III, we show that light cones are in fact (quantum) caustics and hence their natural mathematical description is via catastrophe theory. In Sec. IV, we introduce the XY and TFIM spin chains focusing on the quasiparticle dispersion relation which is the key ingredient we need to apply catastrophe theory. This program is implemented in Sec. V where we obtain the Airy and Pearcey functions for the wave functions dressing the fold and cusp catastrophes/cones in these models. In Sec. VI, we verify the self-similar scaling properties of light cones that catastrophe theory predicts and in Sec. VII, we describe how higher-order catastrophes arise in the context of correlation functions. In Sec. VIII, we identify and discuss the presence of vortex-antivortex pairs within light cones, while in Sec. IX we touch on the relevance of the theory to quench experiments, and in Sec. X we conclude with a discussion of the broader significance of the results. In order to make this paper self-contained, we have included in Appendices A-F the specifics of quantum spin chain diagonalization methods and various other details of our calculations.

\section{GEOMETRIC AND WAVE CATASTROPHES}

In what follows, we will not need the full mathematical machinery behind catastrophe theory, but we will make use of a number of key results and for this reason we give a brief overview here. Our treatment is informal, but we emphasize that these results can be proved rigorously. The main idea can be stated simply: catastrophe theory classifies structurally stable singularities of functions and shows that such singularities can only take on certain characteristic shapes [50]. In up to four dimensions, these are René Thom's seven elementary catastrophes which are listed in Table I.

Each catastrophe arises from two or more coalescing/ bifurcating stationary points of its generating function $\Phi_{Q}$, the normal forms for which are given in the table. In the physical applications given in this paper, $\Phi_{Q}$ is the action functional and stationary points therefore correspond to classical paths or rays. From an optical/classical mechanics point of view a catastrophe is a caustic, i.e., the locus of points where the ray density diverges.

Thom's theorem states that the local behavior of a function near coalescing stationary points can always be mapped by a smooth change of variables onto one of the catastrophes and in this sense catastrophes are universal. There is also a second sense in which catastrophes are universal: structural stability means stability against perturbations and thus catastrophes do not require special symmetry and hence occur generically in nature. Perturbations do not qualitatively change catastrophes and only quantitatively affect behavior up to the strength of the perturbation.

The catastrophes in Table I are organized by the number $n$ of state variables (their corank), and by the dimension $Q$ of the control parameter space. Control space is the space where the function with its singularities actually lives. The control parameters $\mathbf{C}=\left\{C_{1}, C_{2}, \ldots\right\}$ could be space and time coordinates as well as any other parameters. The state variables $\mathbf{s}=\left\{s_{1}, s_{2}, \ldots\right\}$ characterize the rays. The simplest catastrophes (the cuspoids) have $n=1$ and their generating functions are polynomials of the form

$$
\Phi_{Q}(s ; \mathbf{C})=\frac{s^{Q+2}}{Q+2}+\sum_{m=1}^{Q} \frac{C_{m} s^{m}}{m},
$$

with up to $Q$ coalescing stationary points. The stationarity condition reads

$$
\frac{\partial \Phi_{Q}}{\partial s}=0
$$

and corresponds physically to Hamilton's principle of stationary action, while caustics arise from coalescing stationary 
points where the generating function is stationary to higher order [52]

$$
\frac{\partial^{2} \Phi_{Q}}{\partial s^{2}}=0
$$

In the examples, we provide in subsequent sections, we focus primarily on the fold and cusp catastrophes, as well as a discussion of the hyperbolic umbilic in the context of correlation functions. Folds and cusps are the only structurally stable singularities in the $2 \mathrm{D}(x, t)$ control plane where light cones in 1D chains live, while the higher catastrophes (although they may still exist in greater dimensions) can only be projected onto the plane by way of cusps and folds. This property is generic: catastrophes of higher order contain the lower ones [51]. The cusp is the meeting of two fold lines, the swallowtail contains two cusps, and so on.

The wave functions, or wave catastrophes, associated with catastrophes can be obtained in a way analogous to Feynman path integrals by exponentiating the generating function and integrating over all paths,

$$
\Psi_{Q}(\mathbf{C}) \propto \lambda^{n / 2} \int_{-\infty}^{\infty} \cdots \int_{-\infty}^{\infty} d^{n} s e^{\mathrm{i} \lambda \Phi_{Q}(\mathbf{s} ; \mathbf{C})},
$$

where $\lambda$ plays the role of the wave number $k$ or $1 / \hbar$ in quantum problems. In this form, the fact that the generating function plays the role of the physical action becomes clear. These functions are also known as diffraction integrals and many of their properties have been tabulated [44]. We emphasize that standard approximations such as the method of stationary phase where the integral over $\mathbf{s}$ is broken up into a sum of independent gaussian integrals around each of the stationary points are doomed to failure when the stationary points coalesce. One must instead keep the full form of $\Phi_{Q}$ to get a result which is uniformly correct through the coalescence regions and this is precisely why diffraction integrals are crucial for treating bifurcation problems where solutions appear or disappear.

The fold has a cubic action $\Phi_{1}(s ; C)=s^{3} / 3+C s$, where in the case of a light cone in $(1+1)$ dimensions $C=C(x, t)$. As the control parameter $C$ is taken from positive values down through zero the cubic changes its form so as to describe two coalescing rays. The resulting wave catastrophe can be recognized as the integral form of the Airy function,

$$
\Psi_{1}(C) \propto\left(2 \pi \lambda^{1 / 6}\right) \operatorname{Ai}\left(\lambda^{2 / 3} C\right) .
$$

In the absence of any special symmetry, two fold lines generically meet at cusps. In the region near the cusp point the appropriate action is quartic and features two control parameters $\Phi_{2}\left(s ; C_{1}, C_{2}\right)=s^{4} / 4+C_{2} s^{2} / 2+C_{1} s$. This normal form, which formally resembles the Landau free energy for a continuous (second-order) phase transition, describes the coalescence of up to three rays and results in a wave catastrophe known as the Pearcey function,

$$
\Psi_{2}\left(C_{1}, C_{2}\right) \propto\left(2 \pi \lambda^{1 / 4}\right) \operatorname{Pe}\left(C_{1} \lambda^{3 / 4}, C_{2} \lambda^{1 / 2}\right),
$$

which is a complex function of two variables. For our definitions/conventions for the Airy and Pearcey functions, see Eqs. (D5) and (C7), respectively. Plots of the absolute values $|\operatorname{Ai}(C)|$ and $\left|\operatorname{Pe}\left(C_{1}, C_{2}\right)\right|$ of the Airy and Pearcey functions are given in Fig. 1.
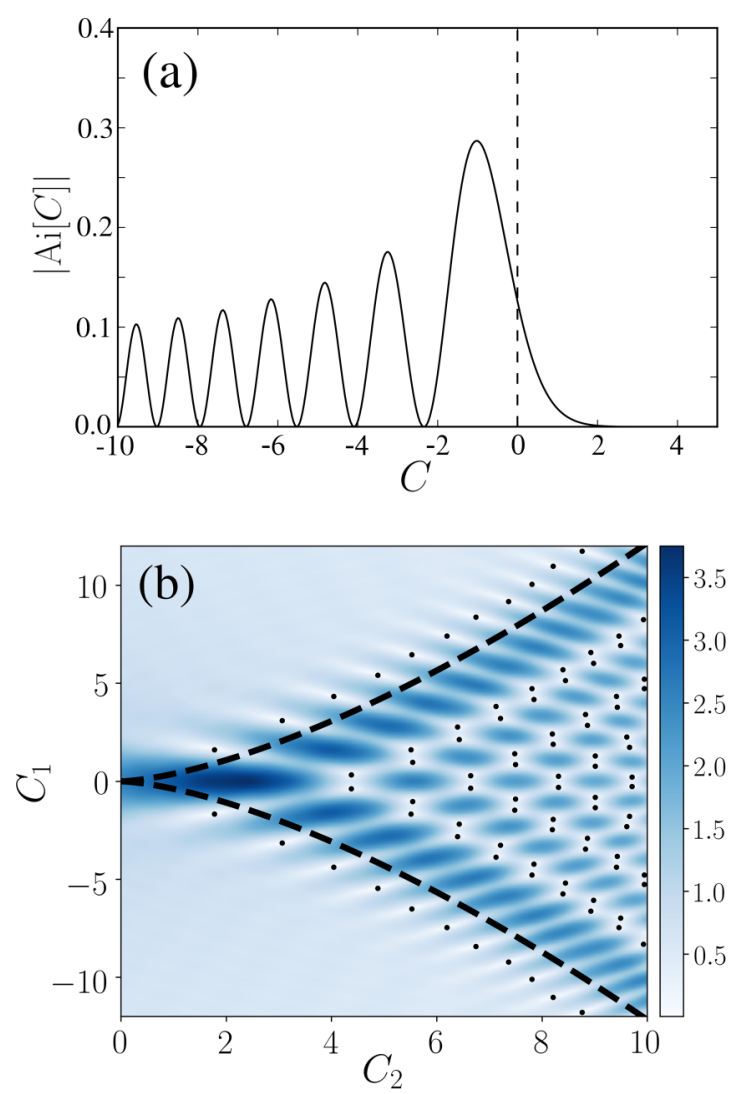

FIG. 1. The Airy and Pearcey functions are the first two wave catastrophes in a hierarchy. (a) Modulus of the Airy function, as defined in Eq. (D5), which dresses a fold catastrophe where two rays coalesce. The location of the fold, or classical caustic, is at $C=0$ and is indicated by the dashed line. For $C<0$ there is two-wave interference giving fringes whereas for $C>0$ there is an evanescent wave. (b) Modulus of the Pearcey function, as defined in Eq. (C7), which dresses the classical cusp caustic $C_{1}=2 C_{2}^{3 / 2} /(3 \sqrt{3})$ and which is shown as a black dashed line. The cusp is made of two fold lines which meet at the cusp tip at $C_{1}=C_{2}=0$. There are three rays/waves inside the cusp and only one outside: two coalesce as we cross either of the fold lines, but all three coalesce at the cusp tip which is the most singular part of the classical caustic (a ray picture of the cusp can be seen in Fig. 2(b) in Ref. [80]). However, wave interference removes the classical singularities. The black dots show the locations of vortices: there is a line of vortices outside either edge of the cusp, and vortex-antivortex pairs inside.

The fact that the Pearcey function is a two-dimensional complex function, with an amplitude and a phase at each point, allows for the possibility of vortices. This turns out to be the case: the black dots in Fig. 1(b) show the locations of vortices, or more precisely their cores. There is an ordered network of vortex-antivortex pairs inside the cusp and single rows of vortices lining the outer edges. These are subwavelength features that represent the finest layer of structure of a wave catastrophe. We find the vortices by densely covering the plane with loops around which we integrate the phase of the Pearcey function: loops that contain vortices give a $\pm 2 \pi$ phase change (the vortex cores also correspond to nodes of the Pearcey function, although in principle not all nodes need be vortices). 
An important feature of wave catastrophes is that they exhibit self-similar scaling. If the parameter $\lambda$ is changed from $\lambda^{\prime}$ to $\lambda$ the wave functions will retain their functional forms but with rescaled coordinates,

$$
\Psi_{Q}\left(\left\{C_{m}\right\} ; \lambda\right)=\left(\frac{\lambda}{\lambda^{\prime}}\right)^{\beta_{Q}} \Psi_{Q}\left(\left\{\left(\frac{\lambda}{\lambda^{\prime}}\right)^{\varsigma_{m}} C_{m}\right\} ; \lambda^{\prime}\right) .
$$

We can understand this scaling as follows: the overall amplitude scales as $\lambda^{\beta_{Q}}$, where $\beta_{Q}$ is known as the Arnol'd index. The distance between interference fringes is also rescaled, but generally the scale factor is different in each direction according to $\lambda^{5 m}$, where $\varsigma_{m}$ is the Berry index associated with coordinate $C_{m}$. For the fold wave catastrophe, $\beta_{\mathrm{Ai}}=\frac{1}{6}$ and $\varsigma=\frac{2}{3}$, and for the cusp wave catastrophe, $\beta_{\mathrm{Pe}}=\frac{1}{4}$ and $\varsigma=\left\{\frac{3}{4}, \frac{1}{2}\right\}$. A complete list of Arnol'd and Berry indices for the seven elementary catastrophes is displayed in Table I.

The sets of Arnol'd and Berry indices accompanying the different catastrophes are reminiscent of the sets of critical exponents which define universality classes of equilibrium phase transitions. The underlying common cause of this similarity is the presence of singularities, or more precisely nonanalyticity, in both cases. We emphasize that in the application to light cones we study here, this universality occurs out of equilibrium, and thus we have an example of universality in quantum dynamics $[79,85,87]$.

\section{LIGHT CONES AS QUANTUM CAUSTICS}

Our approach to the light cone problem is based upon the idea that the build-up of correlations occurs through quasiparticle propagation [3]; this is known to be the case in a broad range of models including the BH, TFIM, and XY models. The Lieb-Robinson bound can then be expressed in terms of the maximal group velocity of quasiparticles [7,9]

$$
v_{\mathrm{LR}}=\max _{k}\left|\frac{d \epsilon_{k}}{d k}\right|,
$$

where $\epsilon_{k}$ is the dispersion relation for quasiparticles as a function of quasimomentum $k$. It can be seen immediately that this result is exactly equivalent to Eqs. (2) and (3) which give the conditions for a caustic (note that here we are implicitly considering real solutions to the caustic conditions; imaginary solutions correspond to phase velocity across the cone and are discussed in Appendix D. This aspect has also been discussed by Cevolani et al. in Ref. [16]). From this simple observation, it follows that light cones are caustics and hence the results and insights of catastrophe theory can be applied to them.

Let us focus on the case of a local quench where a single quasiparticle is created at position $x=0$ in the middle of a spin chain (we briefly consider weakly nonlocal superpositions of multiple quasiparticles in Sec. IX, and also in Appendix E). Time evolving the state with the Hamiltonian $H$, the state vector at time $t$ is

$$
|\Psi(t)\rangle=e^{-\mathrm{i} H t / \hbar} b_{x=0}^{\dagger}|0\rangle_{b}
$$

where $|0\rangle_{b}$ is the Bogoliubov quasiparticle ground state and the operator $b_{x}^{\dagger}$ creates a quasiparticle at the site located at position $x$. For the remainder of the paper, we use the subscript " $b$ " to distinguish Fock states in the Bogoliubov basis from the
Jordan-Wigner basis. Introducing the eigenstates $|k\rangle$ of $H$ we can write this as (see Appendix A for details)

$$
|\Psi(t)\rangle=\frac{e^{\mathrm{i} \theta(t)}}{\sqrt{N}} \sum_{k} e^{-\mathrm{i} \epsilon_{k} t / \hbar}|k\rangle_{b},
$$

where $N$ is the number of sites, and the phase $\theta(t) \equiv$ $t /(2 \hbar) \sum_{k} \epsilon_{k}$ is not observable but is included here for completeness. Projecting onto the position basis, the wave function $\Psi\left(x_{n}, t\right) \equiv\left\langle x_{n} \mid \Psi(t)\right\rangle$ on the $n$th lattice site is

$$
\Psi\left(x_{n}, t\right)=\frac{e^{\mathrm{i} \theta(t)}}{N} \sum_{k_{m}=-\pi / a}^{\pi / a-\Delta k} e^{\mathrm{i} \Phi\left(k_{m} ; x_{n}, t\right)},
$$

where

$$
\Phi(k ; x, t)=k x-\epsilon_{k} t / \hbar .
$$

In these expressions, $n$ is an integer lying in the range $\{-(N-1) / 2, \ldots,(N-1) / 2\}$, and the separation between momenta in the sum is $\Delta k=2 \pi /(a N)$.

In the continuum approximation (CA), the wave function corresponding to Eq. (11) is (see Appendix A)

$$
\Psi_{\mathrm{CA}}(x, t)=\frac{\sqrt{a} e^{\mathrm{i} \theta(t)}}{2 \pi} \int_{-\pi / a}^{\pi / a} d k e^{\mathrm{i} \Phi(k ; x, t)},
$$

where $a=L / N$ is the lattice constant for a lattice of length $L$, and the quasimomentum $k$ runs over the first Brillouin zone. A comparison of the exact (discrete) and CA wave functions is given in Fig. 8 in the Appendices. In the semiclassical regime, where $N$ is large, the dominant contributions to the integral in Eq. (13) come from values of $k$ where $\Phi$ is slowly varying which are the stationary and coalescence points (especially the latter). By Thom's theorem [50-52], we can therefore map $\Phi$ onto one of the normal forms $\Phi_{Q}$. However, although Thom's theorem guarantees that this can be done by smooth transformations, it does not tell us what these transformations actually are. Figuring out the mapping is part of the challenge in applying catastrophe theory to specific physical problems and it is to this task that we now turn.

\section{XY AND TFIM SPIN CHAINS}

Let us consider a 1D XY model describing spins on a lattice interacting with a ferromagnetic coupling $J$, anisotropy parameter $\gamma$, and subject to an external field $g J$. The Hamiltonian is

$$
H=-J \sum_{i}\left(\frac{(1+\gamma)}{2} \sigma_{i}^{x} \sigma_{i+1}^{x}+\frac{(1-\gamma)}{2} \sigma_{i}^{y} \sigma_{i+1}^{y}-g \sigma_{i}^{z}\right),
$$

where $\sigma_{i}^{\alpha}, \alpha \in\{x, y, z\}$, are Pauli operators. When $\gamma=1$ this Hamiltonian reduces to that of the TFIM. The XY Hamiltonian can be diagonalized via the Jordan-Wigner transform followed by a Bogoliubov rotation, which maps spin operators to spinless fermions [82]. As shown in Appendix B, this leads to the free model $H=\sum_{k} \epsilon_{k}\left(\tilde{b}_{k}^{\dagger} \tilde{b}_{k}-1 / 2\right)$, where $\tilde{b}_{k}^{(\dagger)}$ is the annihilation (creation) operator for Bogoliubov modes with quasimomentum $k$ and dispersion

$$
\epsilon_{k}=2 J \sqrt{(\cos (k a)-g)^{2}+\gamma^{2} \sin ^{2}(k a)} .
$$



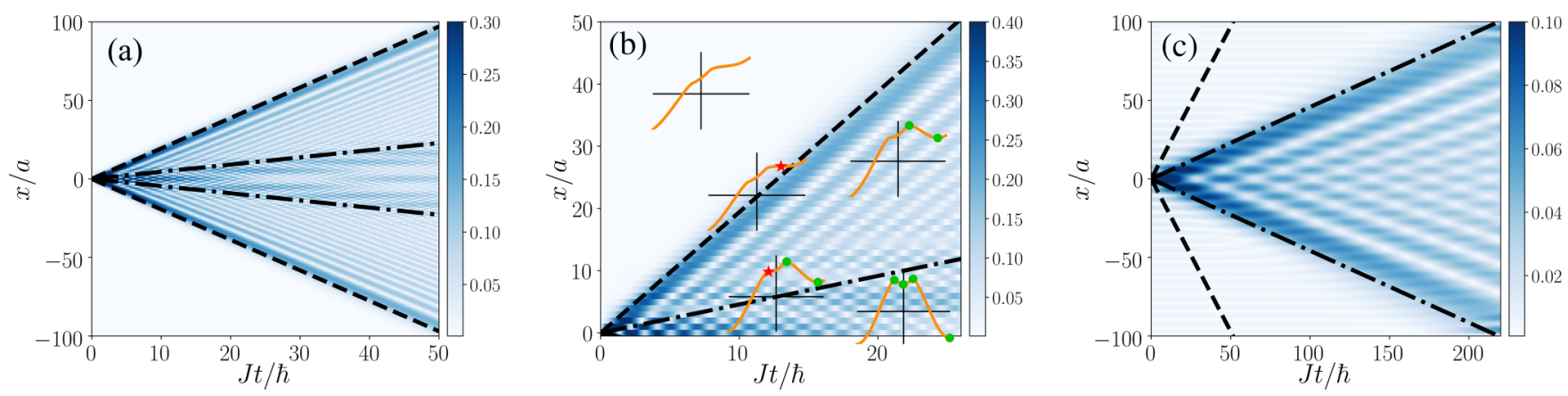

FIG. 2. (a) The exact quantum amplitude, obtained by numerically evaluating Eq. (11), for a single Bogoliubov fermion created at the central lattice site, $x=0$, and propagated under the XY Hamiltonian with $\gamma=0.2$ and $g=0.8$. This model gives rise to a double light cone whose boundaries are indicated by the black dashed (LR cone) and dot-dashed (inner cone) lines. (b) A zoom-in of (a) with only half the lattice shown. At five select points $\left(x_{n}, t\right)$, we have overlaid plots of the generating function $\Phi\left(k ; x_{n}, t\right)$ [Eq. (16)] as a function of $k$. Green dots show stationary points of $\Phi\left(k ; x_{n}, t\right)$; there are four stationary points in the inner cone and two annihilate (red stars) each time we cross a cone boundary. (c) We can isolate the part of $\Psi$ responsible for the inner cone by only including values of $k_{m}$ in Eq. (11) that include the three stationary points of $\Phi$ that are close to the center of the Brillouin zone (note also the change in timescale). As shown in Sec. V, the inner cone is described by a Pearcey function transformed so as to give a straight cone boundary. Note that in order to keep these figures simple we have not shown the vortices although they are present. See Fig. 3 below, and also Fig. 8 in Appendices, for plots of light cone wave functions with vortices included.

Thus the phase/generating function in Eq. (12) takes the specific form

$$
\Phi(k ; x, t)=k x-\frac{2 J t}{\hbar} \sqrt{(\cos (k a)-g)^{2}+\gamma^{2} \sin ^{2}(k a)} .
$$

An exact numerical evaluation of the wave function given in Eq. (11) using the generating function $\Phi(k ; x, t)$ for the $\mathrm{XY}$ model is plotted in Fig. 2. The fact that $x_{n}$ is discrete means that the light cone actually corresponds to a quantum catastrophe, for more discussion of quantum catastrophes in a spin context see Ref. [80]. However, in the semiclassical regime where $N$ is large, the CA described by Eq. (13) works well. In this case, $\Phi$ has the same functional form but with $x$ and $k$ taken as continuous variables, and the integral can be evaluated analytically in terms of the Airy and Pearcey functions as will be explained in the next section.

Dividing $\Phi(k, x, t)$ as given in Eq. (16) by $t$ we can identify three control parameters: $(x / t, \gamma, g)$ [we reserve the energy scale $J$ to play the role of $k$ in Eq. (4)]. However, rays propagate in the $2 \mathrm{D}(x, t)$ plane rather than the full 3D control space and thus for generic values of the control parameters catastrophe theory predicts we should see folds and cusps. In fact, we find a double cone made of a cusp enclosed by two folds as shown in Fig. 2 (double cones occur both in spin systems and in coupled 1D gases [57,58]).

Mathematically speaking, the double cone arises because Eq. (16) has up to four stationary points within the first Brillouin zone, as shown by the green dots in the five overlays plotted in Fig. 2(b). Near the origin in Fig. 2 all four stationary points are present, but three are quasidegenerate so $\Psi$ is locally dominated by a Pearcey-like function, which gives the inner cone. As we cross the edges of the inner cone two stationary points annihilate (indicated by red stars in the overlays) leaving two rays which in turn annihilate at the edges of the outer cone so that locally it is dominated by the Airy function. Furthermore, the XY model has a QCP at $g=1-\gamma^{2}$; as the critical regime is approached the inner cone narrows and eventually collapses because the three inner stationary points in the generating function coalesce at this value of $g$. In the case of the TFIM $(\gamma=1)[4,83,84], \Phi$ has only two stationary points and one finds a single cone with edges that are dressed by Airy functions. The insight from catastrophe theory is that the single cone is nongeneric and only occurs due to the special symmetry of the Hamiltonian when $\gamma=1$.

Due to the presence of four stationary points, the careful reader might expect the $\mathrm{XY}$ model to show signatures of the swallowtail catastrophe. Indeed, this would generically be true, however it can be verified that the quadruple root coalescence do not occur for real $k$. It is the periodic dispersion relation of the model which keeps us from physically probing the highly singular swallowtail point. The cusp and fold catastrophes that we observe here are however inherited from the part of the swallowtail which is physically permitted.

\section{AIRY AND PEARCEY FUNCTIONS}

Let us now demonstrate explicitly how the Airy and Pearcey catastrophe integrals emerge in the CA. Starting with the Pearcey integral, consider first the triple stationary point coalescence responsible for the inner cone, which we have isolated in Fig. 2(c). One obvious difference between this wave function and the Pearcey function shown in Fig. 1 is that the cone boundary in the former is straight rather than the standard curved form of the cusp $C_{1}= \pm \sqrt{4 C_{2}^{3} / 27}$. Physically, this is due to the free propagation of the fermionic quasiparticles. The required transformation to take us between physical coordinates and those of the standard curved cusp is similar to that used by Kaminski and Paris in Ref. [90]. In Appendix C, we show that for our spin model it is

$$
\begin{gathered}
C_{1}=-\sqrt{2} x /\left[v_{\mathrm{I}}(t \Gamma)^{\frac{1}{4}}\right], \\
C_{2}=-\sqrt{t}\left(\gamma^{2}+g-1\right) /[\sqrt{\Gamma}(g-1)],
\end{gathered}
$$



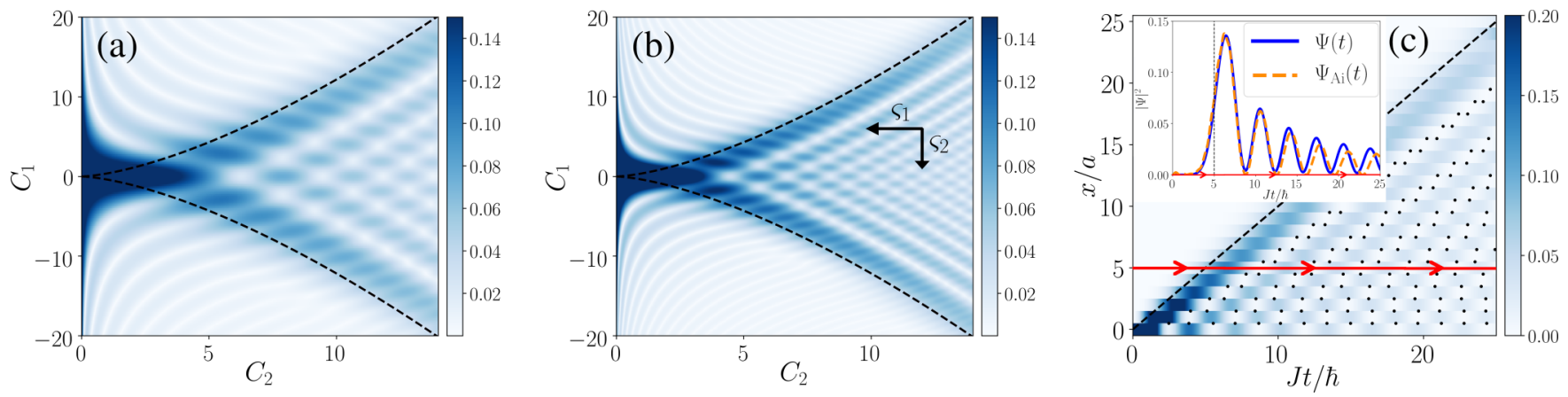

FIG. 3. (a) and (b) Modulus of exact wave function (11) $\Psi\left[x\left(C_{1}, C_{2}\right), t\left(C_{1}, C_{2}\right)\right]$ plotted in the scaled coordinates for the inner cone only (vortices are present but not shown). It shows a remarkable qualitative resemblance to the Pearcey function (compare with Fig. 1), without performing any approximations. Between (a) and (b), the interaction strength has been changed by a ratio of $J^{\prime} / J=2$, so that while the classical ray caustic remains fixed $\left(C_{1}= \pm \sqrt{4 C_{2}^{3} / 27}\right.$, red dashed line), the interference fringes of the wave-function scale according to the Berry indices in the directions indicated. (c) $|\Psi|^{2}$ for the TFIM (blue shading truncated at 0.2 for clarity) is enclosed by the light cone (black, dashed). Black dots mark the locations of vortices (see Sec. VIII). Slice along the time axis at $x / a=5$. The local structure of the exact wave function (11) (blue, solid) near the light cone is well-captured by the Airy-like representation of the wave function (21) (orange, dashed). Away from the caustic the Airy function approximation gradually moves out of the phase with the exact result. This is because we have expanded the generating function about the caustic and can be corrected by performing a uniform approximation.

where $\Gamma=\frac{\left(g^{3}-1-2 \gamma^{2}+3 \gamma^{4}+g\left(3-2 \gamma^{2}\right)+g^{2}\left(4 \gamma^{2}-3\right)\right)}{12(g-1)^{3}}$ and we have defined the Ising velocity,

$$
v_{\mathrm{I}} \equiv\left\{\begin{array}{ll}
\frac{2 J a g}{\hbar} & 0<|g|<1 \\
\frac{2 J a}{\hbar} & 1<|g|
\end{array},\right.
$$

which is equal to $v_{\mathrm{LR}}$ in the TFIM limit (in principle, $v_{\mathrm{LR}}$ can be analytically solved for in closed form for general $\gamma$, however, the expression is complicated, and little physical insight is gained from writing it here).

To complete the diffraction integral we also need the integration variable $s$. This reads $s=\sqrt{2} a(t \Gamma)^{\frac{1}{4}} k$ and results in the Pearcey-like wave function $\Psi_{\mathrm{Pe}}\left(C_{1}, C_{2} ; J\right)$ written out in Eq. (20). It rapidly tends to a true Pearcey function at longer times when $S=\sqrt{2} \pi(t \Gamma)^{\frac{1}{4}} \gg 1$.

$$
\begin{gathered}
\Psi_{\mathrm{Pe}}\left(C_{1}, C_{2} ; J\right) \approx \frac{1}{2 \pi}\left(\frac{J\left(\gamma^{2}+g-1\right)}{\hbar v_{\mathrm{I}}(g-1) C_{2}}\right)^{\frac{1}{2}} \int_{-S}^{S} d s e^{-\frac{\mathrm{i} J}{\hbar} \Phi_{2}\left(s ; C_{1}, C_{2}\right)} \stackrel{J t / \hbar \gg 1}{\propto}\left(\frac{J}{\hbar}\right)^{\frac{1}{4}} \operatorname{Pe}\left[\left(\frac{J}{\hbar}\right)^{\frac{3}{4}} C_{1},\left(\frac{J}{\hbar}\right)^{\frac{1}{2}} C_{2}\right], \\
\Psi_{\mathrm{Ai}}^{\gamma=1}\left(C^{j} ; J\right) \approx \frac{1}{2 \pi t^{1 / 3}}\left(\frac{2 J g^{\frac{2-j}{3}}}{v_{\mathrm{I}} \hbar}\right)^{\frac{1}{2}} \int_{s_{j}^{\mathrm{M}}}^{s_{j}^{\mathrm{Max}}} d s_{j} e^{\frac{\mathrm{i} J}{\hbar} \Phi_{1}\left(s_{j} ; C^{j}\right)} \stackrel{J t / \hbar \gg 1}{\propto}\left(\frac{J}{\hbar}\right)^{\frac{1}{6}} \operatorname{Ai}\left[\left(\frac{J}{\hbar}\right)^{\frac{2}{3}} C^{j}\right] .
\end{gathered}
$$

In order to display the close resemblance between $\Psi_{\mathrm{Pe}}$ and the Pearcey function, we have plotted in Fig. 3 the wave function of the inner cone from Eq. (11) without expansions or approximations in terms of the transformed coordinates $C_{1}$ and $C_{2}$. This can be compared with the actual Pearcey function plotted in Fig. 1. The only significant deviation is near $C_{2}=0$. Since the limit of integration $S$ tends to 0 as $t \rightarrow 0$, the cusp point itself becomes poorly defined, and we get a "smearing" of the wave function as $C_{2} \rightarrow 0$. As a consequence, we cannot get a Pearcey function exactly at the origin, since the initial boundary condition requires the real-space wave function be entirely localized here. As we move away from the cusp point, however, the Pearcey function is indeed an excellent approximation to the true wave function.

As $C_{2}$ increases the Pearcey function can be approximated by two back-to-back Airy functions as the cusp evolves into two fold lines. Indeed, it is a general property of catastrophes that the higher ones evolve into the lower ones as we move away from the former's most singular points. This provides a rigorous explanation for why the Airy functions, which are the simplest of the hierarchy of wave catastrophes, are commonly encountered in the asymptotics of light cones [8,14,15,59-64].

To examine how the Airy function emerges in the CA, we specialize to $\gamma=1$ (TFIM Hamiltonian). We stress that the choice of $\gamma$ does not affect the presence of the fold catastrophe (and thus Airy functions), only the simplicity of the subsequent calculations. To this end, note that for any $g \neq 1$ it can be readily checked that $\Phi(\gamma=1)$ in Eq. (16) has only two stationary points as a function of $k$. We can therefore map onto the canonical fold generating function $\Phi_{1}(s ; C)$ by expanding $\Phi$ to third order in $s$. In the CA, and up to a global phase, we show in Appendix D that the correct control parameter in this case is

$$
C^{j}(x, t)=2\left(x / v_{\mathrm{I}}-t\right)\left(g^{2-j} / \sqrt{t}\right)^{2 / 3} .
$$

The index $j \in\{1,2\}$ refers to cases $g>1$, and $g<1$, corresponding to above and below the QCP, respectively. The integration variable $s_{j}=\left(g^{2-j} t\right)^{\frac{1}{3}}\left[k a-\arccos \left(g^{3-2 j}\right)\right]$ and integration limits $s_{j}^{\text {Min }}=-\left(g^{2-j} t\right)^{1 / 3}\left[\pi+\arccos \left(g^{3-2 j}\right)\right]$ and $s_{j}^{\mathrm{Max}}=\left(g^{2-j} t\right)^{1 / 3}\left[\pi-\arccos \left(g^{3-2 j}\right)\right]$ are also derived in 
Appendix D. The resulting wave function $\Psi_{\mathrm{Ai}}^{\gamma=1}\left(C^{j} ; J\right)$ is given in Eq. (21).

When $\gamma \neq 1$ this process may be repeated around each fold catastrophe, including for any inner cones, and will result in the emergence of Airy functions with different definitions of the control parameter, $C$. For example, a particular limit of Eq. (21) has been conjectured to give a universal form for the wavefront of out-of-time-ordered correlators (OTOCs) [65-68]. According to catastrophe theory this is no surprise. Furthermore, closer to the "brightest" parts of the OTOC the hierarchy of catastrophes allows for more elaborate structures beyond the Airy function.

\section{SCALING}

The way the spin coupling strength $J$ and the control parameters $\mathbf{C}$ appear in combination on the right hand sides of Eqs. (20) and (21) shows that light cones have nontrivial scaling properties: varying $J$ is equivalent to rescaling the amplitude and coordinates. More specifically, increasing $J$ causes the amplitude to increase at a rate determined by the Arnol'd index, and the interference patterns to oscillate more quickly in space and time at rates determined by the Berry index for each particular direction. The overall picture is that the fringes flow in towards the origin as $J$ is increased and in the (singular) classical limit, which occurs when $J \rightarrow \infty$, all wave structure is pulled into the origin. There are other choices we could have made for the scaling parameter since it need only fill the role of $\lambda$ in Eq. (4): for the TFIM, we could have alternatively chosen $a$ or $g$, and in the case of the XY model we could also have chosen either of these or even $\gamma$. It is usually necessary to keep some physics constant during the scaling: we can keep the position of the classical ray caustics constant as $J$ is varied by tuning $a$ or $g$ to keep $v_{\text {I }}$ unchanged.

Numerical verification of the catastrophe theory predictions for both the Arnol'd and Berry indices for the exact wave function Eq. (11) is presented in Fig. 4. Panels (a)-(c) show the scaling in the inner cone of the XY model: the fringe scaling is obtained by measuring the distance between peaks of the wave function along coordinates $C_{1}$ and $C_{2}$ as $J$ is varied and match the Pearcey scaling given in Table I to within $1 \%$. At first glance, it appears that panel (c) shows a contradiction between the expected amplitude scaling of the catastrophe integral and the wave function. However, a quick calculation involving the prefactor of the wave function which ensures that particle number is conserved shows that

$$
C_{2}^{-\frac{1}{2}} \sim\left(\frac{J}{J^{\prime}}\right)^{-\frac{1}{4}}
$$

which exactly cancels the Arnol'd scaling. This is a peculiarity of our nongeneric initial condition of starting with a completely localized initial state: when tracking a particular fringe, it will move towards the origin but this normalization factor means that its height does not scale with $J$.

Panel (d) of Fig. 4 shows the predictions in the TFIM for the period $T$ of oscillations near the caustic. Data are shown both for the exact wave function, given in Eq. (11), and also the "spin-flip" state $\Psi_{\mathrm{X}}$, given in Eq. (33), which is easier to realize experimentally. Since the Berry index $\varsigma$ for the fold
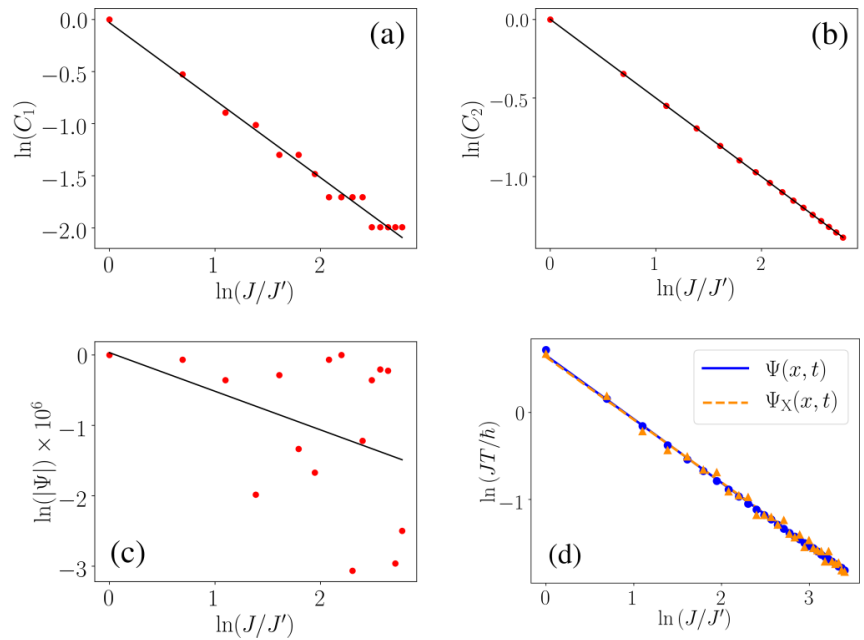

FIG. 4. Self-similar scaling of light cone wave functions. (a) Fringe spacing in the $C_{1}$ direction within the inner cone of the $\mathrm{XY}$ model scales as $J^{\varsigma_{1}}$ with a Berry index $\varsigma_{1} \approx 0.743 \pm 0.002$ (a range of $1 \leqslant J / J^{\prime} \leqslant 16$ was used). The staircase pattern is due to the discreteness of the lattice. (b) Fringe spacing scaling in the $C_{2}$ direction of the XY model gives a Berry index of $\varsigma_{2} \approx$ $0.500 \pm 0.001$. (c) Wave function amplitude scaling of $\ln |\Psi| \approx$ $\left(-6 \times 10^{-7} \pm 4 \times 10^{-7}\right) \ln (J)$, indicating that the amplitude near the cusp has an incredibly weak scaling with $J$. This effect is explained by Eq. (23), since the initial condition precisely cancels the Arnol'd scaling to preserve particle number. (d) The oscillation period, $T$, of $\Psi\left(x_{n}, t\right)$ for site $x / a=5$ in the TFIM with $g=3$; Eqs. (11) and (33) are plotted in blue circles and orange triangles, with blue-solid and orange-dashed trendlines, respectively (a range of $1 \leqslant J / J^{\prime} \leqslant 30$ was chosen). Accounting for a geometric factor of $\sin [\arctan (20)]$, we find the Berry index to be $0.654 \pm 0.003$ and $0.646 \pm 0.009$ for $\Psi$ and $\Psi_{\mathrm{X}}$, respectively.

defines scaling perpendicular to the caustic, a geometric factor dependent on $v_{\mathrm{LR}}$ must be applied. Numerical agreement to within 3\% of Airy scaling given in Table I is found in both cases even for finite-sized systems at finite times.

\section{CORRELATION FUNCTIONS AND HIGHER-ORDER CATASTROPHES}

Rather than the probability distribution associated with the wave function itself, light cones are usually observed in correlation functions [17-21]. The equal time site-site correlation function is defined as

$$
G\left(x_{n}, x_{m}, t\right)=\left\langle b_{n}^{\dagger} b_{m}(t)\right\rangle-\left\langle b_{n}^{\dagger}(t)\right\rangle\left\langle b_{m}(t)\right\rangle .
$$

Because Bogoliubov fermions are conserved, $\left\langle b_{n}^{\dagger}(t)\right\rangle=$ $\left\langle b_{m}(t)\right\rangle=0$, and the last term vanishes. The remaining piece is

$$
\begin{aligned}
\left\langle b_{n}^{\dagger} b_{m}(t)\right\rangle & =\left\langle\Psi(t)\left|b_{n}^{\dagger} b_{m}\right| \Psi(t)\right\rangle \\
& =\frac{1}{N} \sum_{k, k^{\prime}} e^{-\mathrm{i}\left(\epsilon_{k}-\epsilon_{k^{\prime}}\right) t / \hbar}{ }_{b}\left\langle 0\left|\tilde{b}_{k^{\prime}} b_{n}^{\dagger} b_{m} \tilde{b}_{k}^{\dagger}\right| 0\right\rangle_{b} .
\end{aligned}
$$

where we have used the state vector $|\Psi(t)\rangle$ given in Eq. (10). Expressing all the operators in terms of quasimomentum (see 

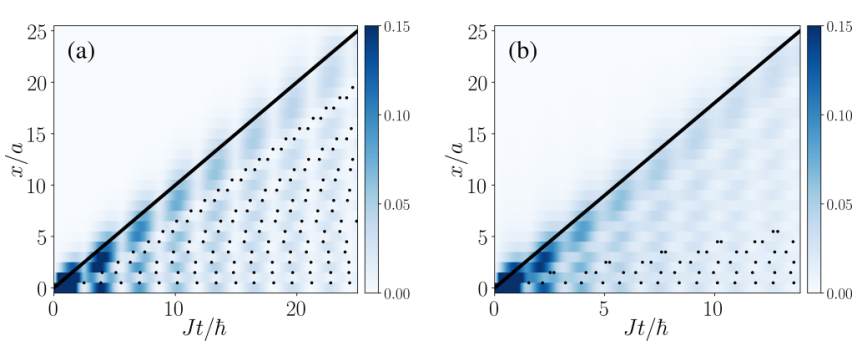

FIG. 5. (a) Correlation function amplitude $\left|G\left(0, x_{m}, t\right)\right|$ for $\gamma=1$, and $g=0.5$, where the blue shading has been truncated at 0.15 for clarity. Black dots indicate vortices. (b) Same correlation function as in (a), now with $g=0.9$. Note that the number of vortices within the cone decreases drastically near the critical point (see Sec. VIII).

Appendix A), we obtain

$$
\begin{aligned}
G\left(x_{n}, x_{m}, t\right) & =\frac{1}{N^{2}} \sum_{k, k^{\prime}} e^{-\mathrm{i}\left(\epsilon_{k}-\epsilon_{k^{\prime}}\right) t / \hbar} e^{\mathrm{i}\left(k x_{m}-k^{\prime} x_{n}\right)} \\
& =\Psi\left(x_{m}, t\right) \Psi\left(-x_{n},-t\right) .
\end{aligned}
$$

In Fig. 5, we plot $G\left(0, x_{m}, t\right)$ on the upper half of the spin chain for two different values of $g$. It displays the same features as the wave function: a light cone, interference fringes, and vortices. In the $\mathrm{CA}$, the equal time site-site correlation function becomes

$$
\begin{aligned}
G_{\mathrm{CA}}\left(x, x^{\prime}, t\right) & =\frac{a}{(2 \pi)^{2}} \int_{-\pi / a}^{\pi / a} \int_{-\pi / a}^{\pi / a} d k d k^{\prime} e^{\mathrm{i}\left(\Phi(k, x)-\Phi\left(k^{\prime}, x^{\prime}\right)\right)} \\
& =\Psi_{\mathrm{CA}}(x, t) \Psi_{\mathrm{CA}}\left(-x^{\prime},-t\right)
\end{aligned}
$$

and expanding around the cone boundaries gives

$$
G_{\mathrm{CA}}\left(x, x^{\prime}, t\right) \approx \Psi_{\mathrm{Ai}}(C(x, t), t) \Psi_{\mathrm{Ai}}\left(C\left(-x^{\prime},-t\right),-t\right),
$$

where $C(x, t)$ is the same function of $x$ and $t$ as that given in Eq. (22).

Measurements and calculations (based on doublon and holon quasiparticles) on the $\mathrm{BH}$ model following a quench also find a product of two Airy functions for $G\left(x_{n}, x_{m}, t\right)$ [8,17]. However, referring to Table I, generic dimension three singularities (i.e., two spatial coordinates $x_{n}$ and $x_{m}$, as well as time $t$ ) of corank 2 (i.e., two integration variables, like in the two-site correlation function) are the elliptic umbilic, and hyperbolic umbilic catastrophes. The elliptic umbilic diffraction catastrophe has been studied by Berry, Nye, and Wright [88] via the optics of a triangular water droplet lens, while the hyperbolic umbilic is a direct consequence of the primary coma aberration [86] and has been observed in matter waves using electron microscopy [69]. These catastrophes are generally more complicated than a squared Airy function, however, we note that in a certain plane the hyperbolic umbilic wave catastrophe does indeed reduce to the product of two Airy functions. More precisely, the hyperbolic umbilic wave catastrophe is given by [44]

$$
\Psi_{\mathrm{HU}}(x, y, z)=\lambda \iint_{-\infty}^{+\infty} d s_{1} d s_{2} e^{\mathrm{i} \lambda\left(s_{1}^{3}+s_{2}^{3}+C_{3} s_{1} s_{2}+C_{2} s_{2}+C_{1} s_{1}\right)},
$$

and when $C_{3}=0$ this reduces exactly to

$$
\Psi_{\mathrm{HU}}\left(C_{1}, C_{2}, 0\right)=\frac{4 \pi^{2} \lambda^{\frac{1}{3}}}{3^{\frac{2}{3}}} \mathrm{Ai}\left(\frac{C_{1} \lambda^{\frac{2}{3}}}{3^{\frac{1}{3}}}\right) \mathrm{Ai}\left(\frac{C_{2} \lambda^{\frac{2}{3}}}{3^{\frac{1}{3}}}\right) .
$$

Thus, both the $\mathrm{XY}$ model and the $\mathrm{BH}$ model give rise to a nongeneric special case.

What physical quantity could the $C_{3}$ control parameter represent? Studying the form of $\Psi_{\mathrm{HU}}$ given in Eq. (29) we note that $C_{3}$ controls the coupling between the $s_{1}$ and $s_{2}$ variables which in a spin chain correspond to the two quasimomenta $k$ and $k^{\prime}$. For noninteracting quasiparticles, which is the case for the exactly solvable models considered in this paper, the two quasimomenta are uncoupled and thus $C_{3}$ is zero. Furthermore, the particular regime of the $\mathrm{BH}$ model where Refs. $[8,17]$ obtained a product of Airy functions also corresponds to the free quasiparticle case. It is therefore clear that $C_{3}$ can be used to parametrize quasiparticle-quasiparticle scattering, and we predict that a model with interacting quasiparticles will give rise to light cones that sample hyperbolic umbilic wave catastrophes. This feature could be verified in an experiment where the strength of the coupling is varied for then the scaling along $C_{3}$ should go as $\zeta_{3}=1 / 3$.

Other quantities, for example, the spin-spin correlation function, $\Sigma_{n m}=\left\langle\sigma_{n}^{x} \sigma_{m}^{x}\right\rangle-\left\langle\sigma_{n}^{x}\right\rangle\left\langle\sigma_{m}^{x}\right\rangle$, may also be calculated exactly via the Jordan-Wigner and Bogoliubov transformations, and simplified using Wick's theorem. The functional forms of these quantities in the continuum approximation remain diffraction integrals, and thus will also display universal behavior corresponding to catastrophes.

\section{VORTICES AND CRITICALITY}

As seen in Figs. 3 and 5, and also Fig. 8 in the Appendices, we find that light cones contain lattices of vortexantivortex pairs. Vortices form the fine structure of wave catastrophes [86,89-91], and in a continuum are zeros of $\Psi$ where the phase $\chi \equiv \operatorname{Arg} \Psi$ is undefined (takes all values) and has the topological property

$$
\oint_{\mathcal{C}} d \chi= \pm 2 \pi,
$$

where $\mathcal{C}$ is any closed path which contains a single vortex. On a discrete lattice we can still use such circuits to find vortices, but across lattice sites one must perform a sum instead of integrating, meaning that their spatial position is only known up to the lattice constant: in figures we place the vortices between lattice sites. Furthermore, vortices on a lattice need not correspond to nodes or even phase singularities, but to points where the phase difference between adjacent sites is $\pm \pi$ (i.e., phase kinks or dark solitons). Thus, while phase interference regulates the amplitude divergence of ray caustics, the effect of a lattice is to regulate the phase singularities of wave theory. In recent work by some of the authors [80], the regularization of phase singularities by a lattice has been considered in Fock space.

Whereas the classical light cone changes smoothly at the QCP [see, e.g., Eq. (19)], there is a sharp minimum in the vortex density, i.e., many vortex-antivortex pairs annihilate, see Fig. 6. In the CA, all vortices except those closest to 

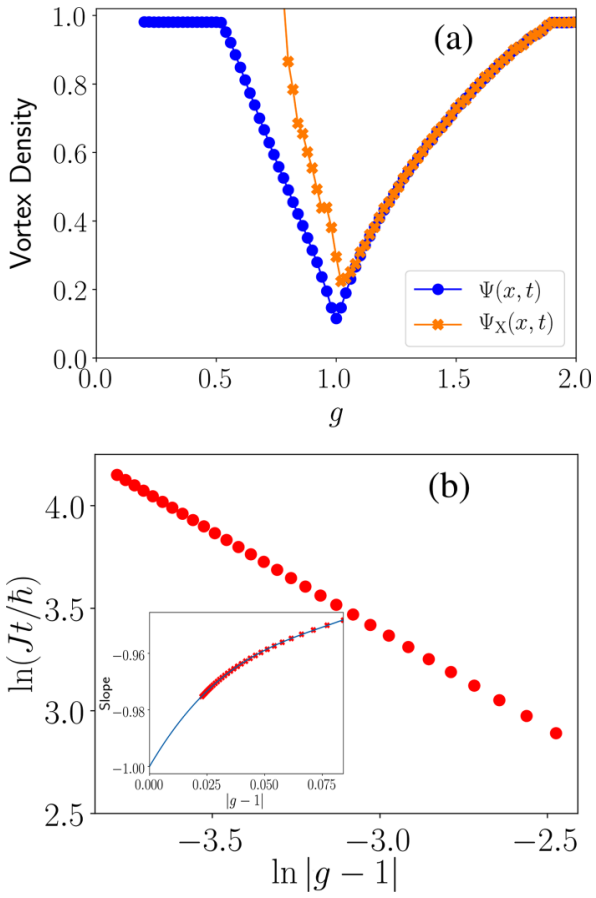

FIG. 6. (a) Vortex density inside the TFIM light cone reaches a sharp minimum at the QCP for both Eq. (11) and the spin-flip state Eq. (33). We define vortex density as being the total number of vortices that occur within a cone up to the time at which the light cone hits the edge of the system, taking care to normalize for different cone sizes at different values of $g$. (b) Numerical determination of vortex pair creation times at a fixed point in space as $g=g_{c}=1$ is approached. In order to extrapolate to the critical point (inset), 30 data points $(g, J t / \hbar)$ are fitted to a quadratic and then differentiated. The resulting slopes are extrapolated to $g_{c}$ using a cubic and the intercept gives $v z=0.9999 \pm 0.0004$ (standard error on the fit). The range $0.02 \leqslant|g-1| \leqslant 0.12$ of $g$ was chosen to optimize the proximity to the critical point along with data accuracy, since the wave function becomes highly oscillatory as $g \rightarrow 1$. Numerical errors are smaller than the symbol sizes.

the central axis annihilate at the QCP, while on a discrete lattice, more off-axis vortices survive but the same trend is observed. At a fixed point in space, the time at which a vortex is first detected increases as one approaches the critical point, becoming infinite in the CA. This diverging timescale $\tau$ is related to critical slowing and suggests a connection to the dynamical critical exponent, $z$. According to the scaling hypothesis of critical phenomena

$$
\tau \propto \xi^{z}
$$

where $\xi=\left|g-g_{c}\right|^{-v}$ is the correlation length and $v$ is its equilibrium critical exponent. Fig. 5(b) plots $\tau$ as found from the wave function Eq. (11) as $g$ is tuned to the QCP. By extrapolating the numerical data [Fig. 5(b) inset] to the critical point we obtain $v z=1$ and hence recover the known critical scaling for the 1D TFIM [92,93]. For purposes of clarity, we have only included the set of vortices which annihilate closest to the axis $x=0$. Vortices which annihilate farther off-axis also display similar trends, which can be seen in Appendix F, along with further figures which help with visualization of this process.
While a more complete understanding of the nature of the vortex-antivortex pairs within the light cone remains a subject of future work, we wish to highlight that their presence and scaling laws provide an interesting link between the predictions of catastrophe theory and universality (in and out of equilibrium). Due to the self-dual nature of the TFIM, qualitative behavior for $g>1$ is identical to that of the wave function below the transition with $g \rightarrow 1 / g$ and $t \rightarrow g t$.

\section{EXPERIMENTAL REALIZATION: SPIN-FLIP STATE}

The structural stability of catastrophes explains why they occur so frequently in nature. Apart from the examples given in the Introduction, they can also occur in disordered systems such as at the Anderson transition where an evanescent Airy function occurs [94], and it has also been shown that wave catastrophes have the property of self-healing after being disrupted [95]. There are, therefore, a broad range of initial conditions and spin models which will give rise to caustics in their dynamics.

So far we have used the initial condition of a localized single quasiparticle, as given in Eq. (9). This is a nongeneric initial condition and the reader may question how generic the resulting light cones really are. In fact, all our analysis is stable to perturbations around this initial condition. In particular, a state which is naturally generated in trapped ion experiments where individual ions can be addressed is a spin-flip state which starts with all spins polarized in the $x$ direction, except for the central spin, say, which is flipped [21],

$$
\Psi_{\mathrm{X}}(x, t) \equiv\left\langle x\left|e^{-\mathrm{i} H t / \hbar}\right| \uparrow^{x} \ldots \uparrow^{x} \downarrow^{x} \uparrow^{x} \ldots \uparrow^{x}\right\rangle .
$$

It is important to realize that physical spins are in general superpositions of multiple quasiparticles and vice versa. We elaborate upon the mathematical details of this point in Appendix $\mathrm{E}$. What we find is that as long as the quench is not too close to the transition the number of quasiparticles created by a spin flip is close to one and hence we are perturbing around the single quasiparticle state given in Eq. (9). The evidence for this statement can be found in Figs. 4(d) and 6(a), which compare the results of using $\Psi_{\mathrm{X}}$ with those of $\Psi$. We find that the scaling properties are essentially identical in the two cases whilst the behavior of the vortex density shows some finite differences but is qualitatively the same.

\section{DISCUSSION AND CONCLUSIONS}

Caustics are a natural phenomenon that can be seen by looking up in the sky on a rainy day. The primary bow of a rainbow is a fold caustic and careful observation reveals supernumerary arcs that are interference fringes described by the Airy function. This is the first in a hierarchy of caustics of increasing complexity whose underlying description is via catastrophe theory. This hierarchy has previously been explored in optics (particularly in the field of gravitational lensing [41]), thermodynamics [96,97], laser physics [98,99], hydrodynamics $[43,45,100]$, and also cosmology $[101,102]$. By showing that light cones in many-body systems are also caustics, we are able to open the door to the application of a rigorous and unified mathematical framework for describing the dynamics of these systems following a quench. 
The main conceptual result of this paper is that there is a hierarchy of light cone structures. They are stable against perturbations and dressed by characteristic wave functions that scale according to the sets of exponents given in Table I. The fold catastrophe and its attendant Airy function features in the TFIM, but breaking the symmetry of the TFIM leads us to the XY model and the second catastrophe, the cusp, which is dressed by the lesser-known Pearcey function. Choosing the spin coupling $J$ as a tuning parameter, we show how the scaling exponents lead to nontrivial scaling of these wave catastrophes as $J$ is varied.

The TFIM and XY models are exactly solvable and hence their quasiparticles are noninteracting. However, the defining feature of catastrophe theory is that it deals with structurally stable singularities and hence the light cone caustics we have described also occur in the presence of perturbations such as weak quasiparticle interactions. A related example of this is provided by the celebrated Kolmogorov-ArnoldMoser (KAM) theorem which shows that tori in the phase space of integrable systems are stable against nonintegrable perturbations. There is in fact a close connection between caustics and the quasiperiodic motion that arises in dynamical systems due to the existence of the tori [103].

Higher-order catastrophes will become important in higher dimension spin lattices. Another way that higher-order catastrophes become important is through $n$-body correlation functions. For the TFIM we find that the two-site equal time correlation function is described near the cone edge by the product of two Airy functions, which is, however, a special case of the hyperbolic umbilic catastrophe. We predict that adding quasiparticle interactions will lead to the full hyperbolic umbilic catastrophe.

On their finest scales, wave catastrophes contain vortexantivortex pairs. We have seen that in the case of light cones in 1D spin chains these become vortex-antivortex pairs in space-time. We note in passing that these are reminiscent of the Kosterlitz-Thouless transition that occurs in one space and one time dimension in the quenched 1D Bose-Hubbard model [104] and in quantum wires [105]. Being high-energy features, we find that the vortices are strongly affected by critical slowing near a QCP, unlike the light cone itself which evolves smoothly. The vortices contain all the information about the QCP and can be used to extract the critical scaling behavior.

The fact that light cones are structurally stable and fall into distinct classes, each of which has its own set of scaling exponents, underlines that as a phenomenon they are an example of universality in out-of-equilibrium dynamics, somewhat akin to the universality classes of equilibrium phase transitions. The underlying reason for this universality in both cases is the presence of singularities, and the realization that light cones are caustics aids us in identifying and understanding their properties.

\section{ACKNOWLEDGMENTS}

We are grateful to Marc Cheneau for first pointing out to us the existence of Airy functions in light cones and to Laurent Sanchez-Palencia for discussions. We acknowledge the support of the Natural Sciences and Engineering Research Council of Canada (NSERC) [Ref. No. RGPIN-2017-06605].

\section{APPENDIX A: DYNAMICS OF A BOGOLIUBOV FERMION}

The spin models dealt with in this paper can be exactly diagonalized in terms of Bogoliubov fermions. Their Hamiltonians can therefore be written in the form

$$
H=\sum_{k} \epsilon_{k}\left(\tilde{b}_{k}^{\dagger} \tilde{b}_{k}-\frac{1}{2}\right)
$$

where $\epsilon_{k}$ is the dispersion relation and the operators $\tilde{b}_{k}^{\dagger}$ and $\tilde{b}_{k}$ create and annihilate, respectively, fermions with quasimomentum $k$. We shall denote the action of the creation operator on the Bogoliubov vacuum as $\tilde{b}_{k}^{\dagger}|0\rangle_{b}=|k\rangle_{b}$. These operators are related to their counterparts in position space via a discrete Fourier transform:

$$
\begin{aligned}
& b_{x}=\frac{1}{\sqrt{N}} \sum_{k} e^{-\mathrm{i} k x} \tilde{b}_{k}, \\
& \tilde{b}_{k}=\frac{1}{\sqrt{N}} \sum_{x} e^{\mathrm{i} k x} b_{x},
\end{aligned}
$$

where $N$ is the number of sites/spins.

Applying the time evolution operator to a single Bogoliubov fermion created at the center of the lattice we obtain the state vector:

$$
\begin{aligned}
|\Psi(t)\rangle & =e^{-\mathrm{i} H t / \hbar} b_{r=0}^{\dagger}|0\rangle_{b}=e^{-\mathrm{i} H t / \hbar}\left(\frac{1}{\sqrt{N}} \sum_{k} \tilde{b}_{k}^{\dagger}\right)|0\rangle_{b} \\
& =\frac{e^{\mathrm{i} \theta(t)}}{\sqrt{N}} \sum_{k} e^{-\mathrm{i} \epsilon_{k} t / \hbar}|k\rangle_{b},
\end{aligned}
$$

where $\theta(t) \equiv(t / 2 \hbar) \sum_{k} \epsilon_{k}$. The corresponding spatial wave function is

$$
\Psi(x, t)={ }_{b}\langle x \mid \Psi(t)\rangle=\frac{e^{\mathrm{i} \theta(t)}}{\sqrt{N}} \sum_{k} e^{-\mathrm{i} \epsilon_{k} t / \hbar} b\langle x \mid k\rangle_{b},
$$

and inserting the standard result $\langle x \mid k\rangle=e^{\mathrm{i} k x} / \sqrt{N}$ for the overlap gives

$$
\Psi(x, t)=\frac{e^{\mathrm{i} \theta(t)}}{N} \sum_{k=-\frac{\pi}{a}}^{\frac{\pi}{a}-\frac{2 \pi}{N a}} e^{\mathrm{i}\left(k x-\epsilon_{k} t / \hbar\right)} .
$$

If we allow $\Delta k=2 \pi /(a N)$ to become very small $(N \gg 1)$ we can approximate the sum by the integral

$$
\Psi(x, t)=\frac{e^{\mathrm{i} \theta(t)} \sqrt{a}}{2 \pi} \int_{-\pi / a}^{\pi / a} d k e^{\mathrm{i} \Phi}
$$

with generating function $\Phi=k x-\frac{t}{\hbar} \epsilon_{k}$. A comparison of the discrete and continuum cases for the TFIM is given in Fig. 8. In the semiclassical regime $(N \gg 1)$, both the sum and the integral are dominated by the points at which $\Phi$ is stationary. Along the caustic, however, a saddle-point approximation fails since we are at a degenerate stationary point. 


\section{APPENDIX B: DIAGONALIZATION OF THE XY MODEL}

The Hamiltonian for the XY model is

$$
H=-\sum_{\langle i j\rangle}\left(J_{x} \sigma_{i}^{x} \sigma_{j}^{x}+J_{y} \sigma_{i}^{y} \sigma_{j}^{y}\right)-h \sum_{i} \sigma_{i}^{z},
$$

where $\sigma_{i}^{\alpha}, \alpha \in\{x, y, z\}$ are the Pauli operators for the $i$ th site. We will use the Jordan-Wigner (JW) transformation, followed by a Bogoliubov rotation, in order to diagonalize $H$. Following the conventions used by Dutta et al. in Ref. [93], the transformation to JW fermions is given by

$$
\begin{gathered}
\sigma_{i}^{z}=2 c_{i}^{\dagger} c_{i}-1, \\
\sigma_{i}^{-}=c_{i} \prod_{j<i}\left(1-2 c_{j}^{\dagger} c_{j}\right)=-\left(c_{i}+c_{i}^{\dagger}\right) e^{\mathrm{i} \pi \sum_{j<i} c_{j}^{\dagger} c_{j}} .
\end{gathered}
$$

We note that the JW fermions and Bogoliubov fermions have different vacuua; some more discussion of this point can be found in Appendix E.

Next, we use a Fourier transform, $\tilde{c}_{k}^{\dagger}=\sum_{j} e^{\mathrm{i} k x_{j}} c_{j}^{\dagger}$, and then rotate to Bogoliubov fermions via

$$
\tilde{b}_{k}^{\dagger}=v_{k} \tilde{c}_{k}+\mathrm{i} u_{k} \tilde{c}_{-k}^{\dagger}
$$

along with the corresponding destruction operator and transformations for $-k$. Here, $u_{k} \equiv \cos \left(\phi_{k} / 2\right), v_{k} \equiv \sin \left(\phi_{k} / 2\right)$, and $\tan \left(\phi_{k}\right)=\left(J_{y}-J_{x}\right) \sin (k a) /\left(\left(J_{y}+J_{x}\right) \cos (k a)+h\right)$, with properties $u_{k}=u_{-k}, v_{k}=-v_{-k}$ in order to ensure the anticommutation relations $\left\{\tilde{c}_{k}^{\dagger}, \tilde{c}_{k^{\prime}}^{\dagger}\right\}=\left\{\tilde{c}_{k}, \tilde{c}_{k^{\prime}}\right\}=0$ and $\left\{\tilde{c}_{k}^{\dagger}, \tilde{c}_{k^{\prime}}\right\}=\delta_{k k^{\prime}}$ hold. We can simplify the resulting Hamiltonian to get it in the form of Eq. (A1) with $\epsilon_{k}=2 \sqrt{h^{2}+J_{x}^{2}+J_{y}^{2}+2 h\left(J_{x}+J_{y}\right) \cos (k a)+2 J_{x} J_{y} \cos (2 k a)}$ being a function of the parameters $J_{x}, J_{y}, h$, and $a$.

Next we introduce the anisotropy parameter $\gamma$ so that we can write $J_{x} \equiv J(1+\gamma) / 2, J_{y} \equiv J(1-\gamma) / 2$ and let $h \equiv g J$. We thereby arrive at the standard form of the Hamiltonian

$$
\frac{H}{J}=-\frac{1}{2} \sum_{\langle i j\rangle}\left((1+\gamma) \sigma_{i}^{x} \sigma_{j}^{x}+(1-\gamma) \sigma_{i}^{y} \sigma_{j}^{y}\right)-g \sum_{i} \sigma_{i}^{z}
$$

with dispersion $\epsilon_{k}=2 J \sqrt{(\cos (k a)+g)^{2}+\gamma^{2} \sin ^{2}(k a)}$. If we change our conventions in order to be consistent with Sachdev [82] we must rotate the Hamiltonian by taking $\sigma^{x} \rightarrow \sigma^{x}, \sigma^{y} \rightarrow \sigma^{y}$, and $\sigma^{z} \rightarrow-\sigma^{z}$. Then we'll instead

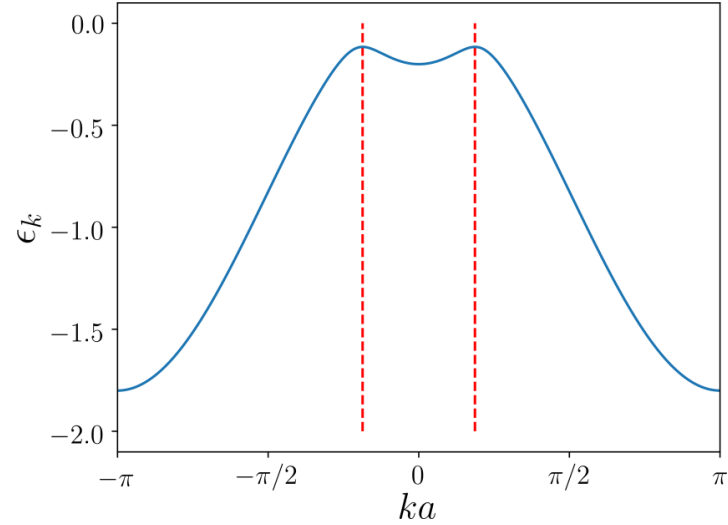

FIG. 7. The XY dispersion relation, given by Eq. (B6), for $\gamma=$ 0.2 and $g=0.8$. Bearing in mind the periodicity of the dispersion relation, one can see that it has four stationary points for these parameter values. The three stationary points that are responsible for the Pearcey function are those that lie between the vertical red dashed lines. The Pearcey function is the wave catastrophe that dresses the inner cone.

have

$$
\epsilon_{k}=2 J \sqrt{(\cos (k a)-g)^{2}+\gamma^{2} \sin ^{2}(k a)} .
$$

Effectively this is like taking $g \rightarrow-g$, allowing us to return to the standard form of the transverse-field Ising model in the $\gamma \rightarrow 1$ limit, presented in the main text. The dispersion relation given in Eq. (B6) is plotted in Fig. 7.

\section{APPENDIX C: CAUSTICS IN THE XY MODEL}

In this Appendix, we give more details of the calculations of the caustics and their wave functions that are presented in the main text. The XY model contains both fold and cusp catastrophes; we focus particularly on the cusp catastrophe and defer some of the treatment of the fold catastrophe to the next Appendix (Appendix D) which is on the TFIM.

\section{Calculation of classical caustics in the $X Y$ model}

The light cone conditions, or equivalently the caustic conditions, are given in Eqs. (2) and (3) in the main text. These correspond to vanishing first and second derivatives of the generating function $\Phi=k x-\epsilon_{k} t / \hbar$. The vanishing of the first derivative with respect to $k$ gives the equation

$$
\frac{\partial \Phi}{\partial k}=x-\frac{J t\left(2 a \gamma^{2} \cos (k a) \sin (k a)+2 a(g-\cos (k a)) \sin (k a)\right)}{\hbar \sqrt{(g-\cos (k a))^{2}+\gamma^{2} \sin ^{2}(k a)}}=0
$$

bringing $x$ to one side, multiplying both sides by the denominator and squaring gives

$$
\begin{aligned}
& x^{2} \hbar^{2}\left((g-\cos (k a))^{2}+\gamma^{2} \sin ^{2}(k a)\right), \\
& \quad=4 a^{2} J^{2} t^{2}\left(g+\left(\gamma^{2}-1\right) \cos (k a)\right)^{2} \sin ^{2}(k a) .
\end{aligned}
$$

Replacing $\sin ^{2}(k a)=1-\cos ^{2}(k a)$, putting $z \equiv \cos (k a)$, and collecting as a quartic polynomial gives

$$
\begin{aligned}
0= & \left(\gamma^{2}-1\right)^{2} v_{\mathrm{I}}^{2} t^{2} z^{4}+2\left(\gamma^{2}-1\right) g v_{\mathrm{I}}^{2} t^{2} z^{3}+\left(g^{2} v_{\mathrm{I}}^{2} t^{2}-\left(\gamma^{2}-1\right)^{2} v_{\mathrm{I}}^{2} t^{2}-\gamma^{2} x^{2}+x^{2}\right) z^{2}+\left(-2\left(\gamma^{2}-1\right) g v_{\mathrm{I}}^{2} t^{2}-2 g x^{2}\right) z \\
& +\gamma^{2} x^{2}-g^{2} v_{\mathrm{I}}^{2} t^{2}+g^{2} x^{2},
\end{aligned}
$$


where the Ising velocity $v_{\mathrm{I}}$ was defined in Eq. (19). The light cones correspond to the control parameter values where solutions coincide, that is, the stationary points of this equation.

The vanishing of the second derivative of the generating function gives the equation

$$
\begin{aligned}
\frac{\partial^{2} \Phi}{\partial k^{2}}= & \frac{J t\left(2 a \gamma^{2} \cos (k a) \sin (k a)-2 a(\cos (k a)-g) \sin (k a)\right)^{2}}{2 \hbar\left((\cos (k a)-g)^{2}+\gamma^{2} \sin ^{2}(k a)\right)^{3 / 2}} \\
& -\frac{J t\left(2 a^{2} \gamma^{2} \cos ^{2}(k a)-2 a^{2} \cos (k a)(\cos (k a)-g)\right)}{\hbar \sqrt{(\cos (k a)-g)^{2}+\gamma^{2} \sin ^{2}(k a)}}-\frac{J t\left(2 a^{2} \sin ^{2}(k a)-2 a^{2} \gamma^{2} \sin ^{2}(k a)\right)}{\hbar \sqrt{(\cos (k a)-g)^{2}+\gamma^{2} \sin ^{2}(k a)}}=0 .
\end{aligned}
$$

We now multiply both sides by $2 \hbar\left[\left((\cos (k a)-g)^{2}+\gamma^{2} \sin ^{2}(k a)\right]^{3 / 2} / J t\right.$ and simplify

$$
\begin{aligned}
0= & \frac{1}{2} a^{2}\left[3\left(4 g^{2}+\left(\gamma^{2}-1\right)^{2}\right)-2 g\left(9+4 g^{2}-5 \gamma^{2}\right) \cos (k a)+4\left(\gamma^{4}-1+g^{2}\left(2 \gamma^{2}-3\right)\right) \cos (2 k a)\right. \\
& \left.+6 g\left(\gamma^{2}-1\right) \cos (3 k a)+\left(\gamma^{2}-1\right)^{2} \cos (4 k a)\right] .
\end{aligned}
$$

Next we make the replacements $\cos (2 k a)=2 \cos ^{2}(k a)-1 ; \cos (3 k a)=4 \cos ^{3}(k a)-3 \cos (k a) ; \operatorname{and} \cos (4 k a)=8 \cos ^{4}(k a)-$ $8 \cos ^{2}(k a)+1$. Defining again $z \equiv \cos (k a)$, and dividing both sides by $a^{2} / 2$,

$$
0=8\left(\gamma^{2}-1\right)^{2} z^{4}+24 g\left(\gamma^{2}-1\right) z^{3}-8\left(2 \gamma^{2}\left(\gamma^{2}-1\right)+g^{2}\left(2 \gamma^{2}-3\right)\right) z^{2}-8 g\left(g^{2}+\gamma^{2}\right) z+8 \gamma^{2}\left(g^{2}+\gamma^{2}-1\right) \text {. }
$$

The light cones/caustics correspond to simultaneous solutions of Eqs. (C3) and (C6) and hence correspond to the LiebRobinson (LR) bound which is the solution which maximizes the propagation speed of the quasiparticles.

In the next section, we describe how the triple coalescence of stationary points give rise to the Pearcey function which provides the inner cone in Fig. 2. The three stationary points which coalesce are those between the dashed lines in Fig. 7. For $0<\gamma<1$ and $0<g<1$, this coalescence occurs at $k=0$, thus $z=1$, and Eq. (C6) yields solutions $g=1$ and $g=1-\gamma^{2}$. The $g=1$ solution is highly singular for nonzero anisotropy, while the solution $g=1-\gamma^{2}$ is the key for triple root coalescence.

\section{Diffraction integral for the cusp wave catastrophe}

Let us begin by defining the Pearcey function which is the canonical form of the wave function corresponding to the cusp catastrophe. The definition of the Pearcey function that we use is

$$
\operatorname{Pe}\left(C_{1}, C_{2}\right) \equiv \frac{1}{2 \pi} \int_{-\infty}^{\infty} d s e^{-\mathrm{i}\left(C_{1} s+\frac{C_{2}}{2} s^{2}+\frac{s^{4}}{4}\right)}
$$

It features two parameters $C_{1}$ and $C_{2}$ and is generally a complex function. In fact, the common definition of the Pearcey function is the complex conjugate of (C7), however for our purposes, the above definition is more convenient.

Since the coalescence of extrema in $\Phi$ occurs at $k=0$, we expand to fourth order and factor out $J / \hbar$, which we will later use for scaling,

$$
\begin{aligned}
\Phi(k ; J) \approx & \frac{J}{\hbar}\left[2 t(g-1)+\frac{2 g a x}{v_{\mathrm{I}}} k\right. \\
& \left.+\frac{1}{2} \frac{2 a^{2} t\left(\gamma^{2}+g-1\right)}{(g-1)} k^{2}-\frac{1}{4} 4 a^{4} t \Gamma k^{4}\right],
\end{aligned}
$$

where we have defined the following parameter:

$$
\Gamma \equiv \frac{\left(g^{3}-1-2 \gamma^{2}+3 \gamma^{4}+g\left(3-2 \gamma^{2}\right)+g^{2}\left(4 \gamma^{2}-3\right)\right)}{12(g-1)^{3}} \text {. }
$$

Note that the solution $g=1-\gamma^{2}$ will kill off the quadratic piece.

We now rescale our integration variable

$$
s=\sqrt{2} a(t \Gamma)^{\frac{1}{4}} k,
$$

then our wave function locally takes the form

$$
\begin{aligned}
& \Psi_{\mathrm{Pe}}\left(C_{1}, C_{2} ; J\right) \\
& \approx \frac{e^{\mathrm{i} \theta(t)}}{2 \pi} \sqrt{\frac{J}{\hbar v_{\mathrm{I}}}}\left(\frac{\gamma^{2}+g-1}{(g-1) C_{2}}\right)^{\frac{1}{2}} \int_{-S}^{S} d s e^{-\mathrm{i} \frac{J}{\hbar}\left(C_{1} s+\frac{C_{2}}{2} s^{2}+\frac{s^{4}}{4}\right)}
\end{aligned}
$$

with definitions

$$
\begin{gathered}
C_{1}(g, \gamma ; x, t)=-\frac{\sqrt{2} x}{v_{\mathrm{I}}(t \Gamma)^{\frac{1}{4}}}, \\
C_{2}(g, \gamma ; x, t)=-\frac{\gamma^{2}+g-1}{g-1}\left(\frac{t}{\Gamma}\right)^{\frac{1}{2}}
\end{gathered}
$$

and integration limit

$$
S=\sqrt{2} \pi(t \Gamma)^{\frac{1}{4}} .
$$

Equation (C11) shows that the wave function for the inner cone can locally be expressed as a diffraction integral which is generated by the cusp catastrophe $\Phi_{2}=C_{1} s+C_{2} s^{2} / 2+s^{4}$, and is thus directly related to the canonical Pearcey function when $t$ is reasonably large and $J / \hbar=1$ (below we will see that we can choose any value of $J / \hbar$ and it will simply rescale the coordinates). Note, however, that the normalization of the wave function restricts the bounds of the integral as $t \rightarrow 0$, and so no true cusp point can occur at the origin since $\Phi$ also vanishes there. Nevertheless, the region of integration is proportional to $t^{1 / 4}$ and so is larger than the separation between the stationary points as $t \rightarrow 0$ since for any quartic equation of the form $\Phi_{2}$ the position of the stationary points in the $s$ coordinate is proportional to $\sqrt{C_{2}}$ so that for any infinitesimal time $d t$ the separation between them is proportional to only $(d t)^{1 / 2}$. Thus it becomes imperative that we 

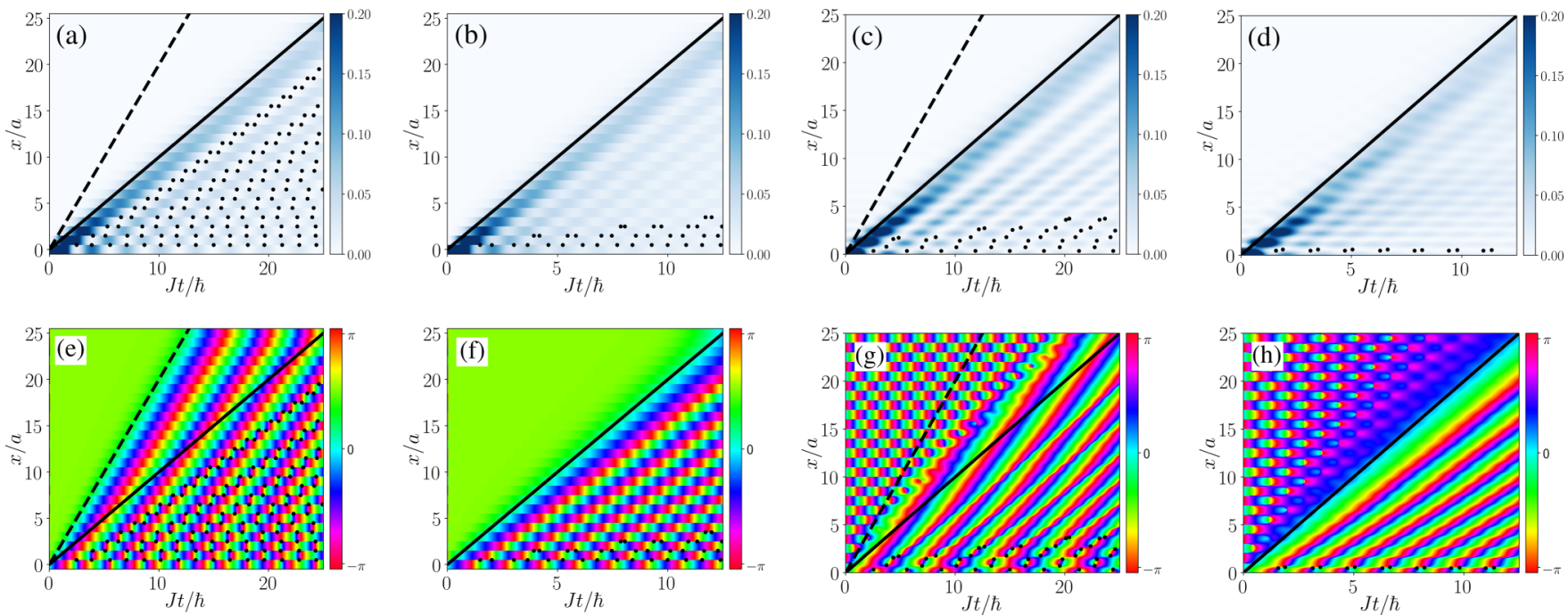

FIG. 8. Caustics and vortices in the TFIM: discrete (exact) versus continuum approximation. The initial condition is a single Bogoliubov fermion created at the center of the chain (only half the chain is shown). The discrete wave function is given by Eq. (A7) whereas the continuum approximation is given by Eq. (A8). [(a)-(d)] Amplitude of the wave function for $g=0.5$ and 1. [(e)-(h)] Phase of the same wave functions (corresponding to the panels directly above). The caustics are shown as solid black lines, while the imaginary caustics are plotted as dashed lines. The black dots mark the locations of vortices.

consider the effects of all three stationary points, giving rise to the Pearcey-like function described in Eq. (20).

Finally, in order to keep our expressions consistent for $|g|<1$ and $|g|>1$, we can instead factor out $J g / \hbar$ overall. The above results are then identical up to a factor of $1 / g$, which can be absorbed into $s$ and is irrelevant for the scaling. Thus the expression $v_{\text {I }}$ given in Eq. (19) may be used in Eq. (C12) generally.

\section{Self-similar scaling of the cusp wave catastrophe}

Now we scale the coupling strength, which corresponds to the width of the dispersion relation, from $J \rightarrow J^{\prime}$. As we do so we enforce $J s^{4}=J^{\prime} s^{\prime 4}$ so that the wave function maintains its basic form. Then, the Berry scaling is

$$
J C_{1} s=J C_{1}\left(\frac{J^{\prime}}{J}\right)^{\frac{1}{4}} s^{\prime}=J^{\prime} C_{1}\left(\frac{J}{J^{\prime}}\right)^{\frac{3}{4}} s^{\prime}
$$

and

$$
J \frac{C_{2}}{2} s^{2}=J \frac{C_{2}}{2}\left(\frac{J^{\prime}}{J}\right)^{\frac{1}{2}} s^{\prime 2}=J^{\prime} \frac{C_{2}}{2}\left(\frac{J}{J^{\prime}}\right)^{\frac{1}{2}} s^{\prime 2}
$$

with Arnol'd scaling given by

$$
\sqrt{J} d s=\sqrt{J}\left(\frac{J^{\prime}}{J}\right)^{\frac{1}{4}} d s^{\prime}=\sqrt{J^{\prime}}\left(\frac{J}{J^{\prime}}\right)^{\frac{1}{4}} d s^{\prime} .
$$

These are the scaling factors for the cusp wave catastrophe as listed in Table I. As we tune $J$, it is convenient to keep the caustic in the same place. This is done by simultaneously tuning $a$ such that the Ising velocity $v_{\mathrm{I}}$ is constant.

\section{APPENDIX D: CAUSTICS IN THE TRANSVERSE-FIELD ISING MODEL}

As mentioned in the main text, the outer light cone in the $\mathrm{XY}$ model is dominated by its Airy-like behavior because it arises from the coalescence of just two stationary points. Since this also occurs in the simpler TFIM (which is obtained by setting $\gamma=1$ ), we focus on this case here.

\section{Calculation of classical caustics in the TFIM}

As shown above for the cusp catastrophe case, we must first calculate the two caustic conditions

$$
\frac{\partial \Phi}{\partial k}=0=x-\frac{2 a g J t \sin (k a)}{\hbar \sqrt{g^{2}-2 g \cos (k a)+1}}
$$

and

$$
\begin{aligned}
\frac{\partial^{2} \Phi}{\partial k^{2}}=0= & -\frac{2 a^{2} g J t \cos (k a)}{\hbar \sqrt{g^{2}-2 g \cos (k a)+1}} \\
& +\frac{2 a^{2} g^{2} J t \sin ^{2}(k a)}{\hbar\left(g^{2}-2 g \cos (k a)+1\right)^{3 / 2}},
\end{aligned}
$$

which must be simultaneously fulfilled. Rearranging Eq. (D2)

$$
g\left(1-\cos ^{2}(k a)\right)=\cos (k a)\left(g^{2}-2 g \cos (k a)+1\right)
$$

leads to $\cos (k a)=g$ or $\cos (k a)=1 / g$, as expected. Inputting this into Eq. (D1), along with $\sin (k a)=\sqrt{1-g^{2}}$ [or $\sin (k a)=\sqrt{1-1 / g^{2}}$ for $g>1$, we can solve for the LR velocity, which is identical to the Ising velocity we defined in the previous section,

$$
v_{\mathrm{LR}}=v_{\mathrm{I}} .
$$

Although the caustic lines are determined by the real solutions to Eq. (D3), there exist imaginary solutions for which the Lieb-Robinson velocity $g$ designations are reversed. This seems to be responsible for lines of constant phase across the caustic (see Fig. 8). The presence of two separate speeds within the light cone is also demonstrated by Cevolani et al. in Ref. [16]. We term these imaginary solutions as "imaginary caustics." 


\section{Diffraction integral for the fold wave catastrophe}

The canonical wave catastrophe corresponding to the fold catastrophe is the Airy function. The definition of the Airy function that we use is

$$
\operatorname{Ai}(C) \equiv \frac{1}{2 \pi} \int_{-\infty}^{\infty} d s e^{\mathrm{i}\left(C s+s^{3} / 3\right)} .
$$

It features a single parameter $C$ and is a real function if $C$ is real.

The stationary points of $\Phi$ coalesce when $k=$ $(1 / a) \arccos (g)$ for $g<1$ and $k=(1 / a) \arccos (1 / g)$ for $g>1$, respectively. Thus, for each of these cases, we will expand about these particular $k$ values to third order and factoring out $J / \hbar$ overall,

$$
\begin{aligned}
\Phi(k ; J) \approx & \frac{J}{\hbar}\left(-2 t \sqrt{1-g^{2}}+\frac{x \hbar}{a J} \arccos (g)\right) \\
& +\frac{J}{\hbar}\left(\frac{x \hbar}{a J}-2 g t\right)(k a-\arccos (g)) \\
& +\frac{J}{\hbar} \frac{1}{3} g t(k a-\arccos (g))^{3}
\end{aligned}
$$

for $g<1$ and

$$
\begin{aligned}
\Phi(k ; J) \approx & \frac{J}{\hbar}\left(-2 t \sqrt{g^{2}-1}+\frac{x \hbar}{a J} \arccos (1 / g)\right) \\
& +\frac{J}{\hbar}\left(\frac{x \hbar}{a J}-2 t\right)(k a-\arccos (1 / g)) \\
& +\frac{J}{\hbar} \frac{1}{3} t(k a-\arccos (1 / g))^{3},
\end{aligned}
$$

for $g>1$. Of course, the expansion will only capture the behavior of the wave function close to the light cone, however this is our primary objective. Furthermore, we are guaranteed that (up to a smooth change of variables) this cubic form in particular is structurally stable and will capture the qualitative features of $\Phi$. We now rescale our integration variables as

$$
\begin{gathered}
s_{1}^{3}=g t(k a-\arccos (g))^{3}, \\
s_{2}^{3}=t(k a-\arccos (1 / g))^{3} .
\end{gathered}
$$

Thus

$$
\begin{aligned}
\Phi_{\mathrm{Ai}}(s ; J)= & \frac{J}{\hbar}\left[\left(-2 t \sqrt{1-g^{2}}+\frac{2 g x}{v_{\mathrm{I}}} \arccos (g)\right)\right. \\
& \left.+2\left(\frac{x}{v_{\mathrm{I}}}-t\right)\left(\frac{g^{2}}{t}\right)^{\frac{1}{3}} s_{1}+\frac{1}{3} s_{1}^{3}\right]
\end{aligned}
$$

for $g<1$ and

$$
\begin{aligned}
\Phi_{\mathrm{Ai}}(s ; J)= & \frac{J}{\hbar}\left[\left(-2 t \sqrt{g^{2}-1}+\frac{2 x}{v_{\mathrm{I}}} \arccos (1 / g)\right)\right. \\
& \left.+2\left(\frac{x}{v_{\mathrm{I}}}-t\right)\left(\frac{1}{t}\right)^{\frac{1}{3}} s_{2}+\frac{1}{3} s_{2}^{3}\right]
\end{aligned}
$$

for $g>1$.

Now we define the control variable as

$$
C=\left\{\begin{array}{ll}
2\left(\frac{x}{v_{\mathrm{I}}}-t\right)\left(\frac{g^{2}}{t}\right)^{\frac{1}{3}}, & g<1 \\
2\left(\frac{x}{v_{\mathrm{I}}}-t\right)\left(\frac{1}{t}\right)^{\frac{1}{3}}, & g>1
\end{array},\right.
$$

so that

$$
\Psi(C ; J)= \begin{cases}\frac{e^{\Theta_{1}}}{2 \pi(g t)^{\frac{1}{3}} \sqrt{a}} \int_{s^{\operatorname{Min}}}^{s^{\operatorname{Max}}} d s e^{\frac{\mathrm{i} J}{\hbar} \Phi_{1}(C, s)}, & g<1 \\ \frac{e_{2}^{\Theta_{2}}}{2 \pi t^{\frac{1}{3}} \sqrt{a}} \int_{s^{\operatorname{Min}}}^{s^{\operatorname{Max}}} d s e^{\frac{\mathrm{i} J}{\hbar} \Phi_{1}(C, s)}, & g>1\end{cases}
$$

with

$$
\begin{gathered}
\Phi_{1}(C, s)=C s+\frac{1}{3} s^{3}, \\
\frac{1}{\sqrt{a}} d s= \begin{cases}\sqrt{\frac{2 g J}{v_{I} \hbar}} d s, & g<1 \\
\sqrt{\frac{2 J}{v_{I} \hbar}} d s, & g>1\end{cases}
\end{gathered}
$$

and limits

$$
\begin{gathered}
s^{\operatorname{Min}}=\left\{\begin{array}{cc}
(g t)^{\frac{1}{3}}(-\pi-\arccos (g)) & g<1 \\
t^{\frac{1}{3}}(-\pi-\arccos (1 / g)) & g>1
\end{array},\right. \\
s^{\operatorname{Max}}=\left\{\begin{array}{cc}
(g t)^{\frac{1}{3}}(\pi-\arccos (g)) & g<1 \\
t^{\frac{1}{3}}(\pi-\arccos (1 / g)) & g>1
\end{array} .\right.
\end{gathered}
$$

Note that $s^{\text {Min }}<0$ and $s^{\text {Max }}>0$. Thus, if we assume long enough times, then it is reasonable to take these integration limits to plus and minus infinity. We now have a description of the wave function local to the light cone using a fold catastrophe integral, which in the limit of $J / \hbar \rightarrow 1$ will become the Airy integral.

\section{Self-similar scaling of the fold wave catastrophe}

As for the cusp case, we can extract the scaling properties of the fold wave catastrophe by considering the change from $J \rightarrow J^{\prime}$. Under this transformation we assume that $J s^{3}=$ $J^{\prime} s^{\prime 3}$. Then,

$$
J C s=J C\left(\frac{J^{\prime}}{J}\right)^{\frac{1}{3}} s^{\prime}=J^{\prime} C\left(\frac{J}{J^{\prime}}\right)^{\frac{2}{3}} s^{\prime}
$$

and

$$
\sqrt{J} d s=\sqrt{J}\left(\frac{J^{\prime}}{J}\right)^{\frac{1}{3}} d s^{\prime}=\sqrt{J^{\prime}}\left(\frac{J}{J^{\prime}}\right)^{\frac{1}{6}} d s^{\prime} .
$$

Taking the integral limits to infinity (long times)

$$
\Psi_{\mathrm{Ai}}(C ; J) \propto \sqrt{J^{\prime}}\left(\frac{J}{J^{\prime}}\right)^{\frac{1}{6}} \int_{-\infty}^{\infty} d s^{\prime} e^{\mathrm{i} \frac{J^{\prime}}{\hbar}\left(\left(\frac{J}{J^{\prime}}\right)^{\frac{2}{3}} C s^{\prime}+\frac{1}{3} s^{\prime 3}\right)}
$$

or, equivalently,

$$
\Psi_{\mathrm{Ai}}(C ; J)=\left(\frac{J}{J^{\prime}}\right)^{\frac{1}{6}} \Psi_{\mathrm{Ai}}\left(\left[\frac{J}{J^{\prime}}\right]^{\frac{2}{3}} C ; J^{\prime}\right) .
$$

We have therefore obtained the scaling factors for the fold wave catastrophe as listed in Table I.

\section{APPENDIX E: SPIN-FLIP STATE $\Psi_{\mathrm{X}}(x, t)$}

In this paper, we mainly consider an initial state consisting of a single fermionic quasiparticle localized on a particular site. However, in Sec. IX, we instead consider the initial state where all the spins are polarized along the $x$ direction except 
for the central spin which is flipped such that the time evolved wave function is

$$
\Psi_{\mathrm{X}}(x, t) \equiv\left\langle x\left|e^{-\mathrm{i} H t / \hbar}\right| \uparrow^{x} \ldots \uparrow^{x} \downarrow^{x} \uparrow^{x} \ldots \uparrow^{x}\right\rangle .
$$

Because experiments with ions can easily address individual spins, and spins and quasiparticles are not quite the same thing, it important to consider this kind of state.

Evaluating the time evolution of spin chains is generally far simpler in the Bogoliubov basis. However, to introduce physical spins, we begin with the JW basis which is related to the Bogoliubov basis by the Bogoliubov rotation:

$$
\tilde{c}_{k}^{\dagger}=u_{k} \tilde{b}_{k}^{\dagger}-\mathrm{i} v_{k} \tilde{b}_{-k} .
$$

We identify the creation of a JW fermion at the center of the lattice as a spin flip from $\uparrow^{x}$ to $\downarrow^{x}$ via the inverse JW transformation:

$$
c_{j}^{\dagger}=\left(\prod_{i>j} \sigma_{i}^{x}\right) \sigma_{j}^{-}
$$

It is also important to note that the JW and Bogoliubov vacuums are related by

$$
|0\rangle=\prod_{k>0}\left(u_{k}-\mathrm{i} v_{k} \tilde{b}_{k}^{\dagger} \tilde{b}_{-k}^{\dagger}\right)|0\rangle_{b} .
$$

Starting with the center spin $(x=0)$ down,

$$
\begin{aligned}
\left|\Psi_{0}\right\rangle= & c_{x=0}^{\dagger}|0\rangle=\sum_{k_{1}} \tilde{c}_{k_{1}}^{\dagger}|0\rangle \\
= & \sum_{k_{1}}\left(u_{k_{1}} \tilde{b}_{k_{1}}^{\dagger}-\mathrm{i} v_{k_{1}} \tilde{b}_{-k_{1}}\right) \\
& \times \prod_{k_{2}>0}\left(u_{k_{2}}-\mathrm{i} v_{k_{2}} \tilde{b}_{k_{2}}^{\dagger} \tilde{b}_{-k_{2}}^{\dagger}\right)|0\rangle_{b}
\end{aligned}
$$

and using the following relation

$$
\begin{aligned}
\tilde{b}_{k_{1}}^{\dagger} & \prod_{k_{2}>0}\left(u_{k_{2}}-\mathrm{i} v_{k_{2}} \tilde{b}_{k_{2}}^{\dagger} \tilde{b}_{-k_{2}}^{\dagger}\right)|0\rangle_{b} \\
& =u_{k_{1}} \tilde{b}_{k_{1}}^{\dagger} \prod_{k_{2}>0,\left|k_{2}\right| \neq\left|k_{1}\right|}\left(u_{k_{2}}-\mathrm{i} v_{k_{2}} \tilde{b}_{k_{2}}^{\dagger} \tilde{b}_{-k_{2}}^{\dagger}\right)|0\rangle_{b},
\end{aligned}
$$

we get

$$
\left|\Psi_{0}\right\rangle=\sum_{k_{1}} \tilde{b}_{k_{1}}^{\dagger} \prod_{k_{2}>0,\left|k_{2}\right| \neq\left|k_{1}\right|}\left(u_{k_{2}}-\mathrm{i} v_{k_{2}} \tilde{b}_{k_{2}}^{\dagger} \tilde{b}_{-k_{2}}^{\dagger}\right)|0\rangle_{b} .
$$

Next, we evolve in time using a more convenient representation of the time-evolution operator,

$$
e^{-\frac{\mathrm{i} t}{\hbar} \sum_{k} \epsilon_{k}\left(\tilde{b}_{k}^{\dagger} \tilde{b}_{k}-\frac{1}{2}\right)}=e^{\mathrm{i} \theta(t)} \prod_{k}\left[1-\left(1-e^{-\frac{\mathrm{i} t \epsilon_{k}}{\hbar}}\right) \tilde{b}_{k}^{\dagger} \tilde{b}_{k}\right] .
$$

Dropping the global phase factor, and projecting this state onto real space $\Psi\left(x_{i}, t\right)=\left\langle x_{i} \mid \Psi(t)\right\rangle$ using

$$
\begin{aligned}
\left\langle x_{i}\right| & =\langle 0| c_{i} \\
& =\sum_{k_{3}} e^{\mathrm{i} k_{3} x_{i}}{ }_{b}\langle 0| \prod_{\substack{k_{4}>0 \\
\left|k_{4}\right| \neq\left|k_{3}\right|}}\left(u_{k_{4}}+\mathrm{i} v_{k_{4}} \tilde{b}_{-k_{4}} \tilde{b}_{k_{4}}\right) \tilde{b}_{k_{3}},
\end{aligned}
$$

we arrive at, after a fair amount of algebra,

$$
\begin{aligned}
\Psi\left(x_{i}, t\right)= & \sum_{k_{1}} e^{-\frac{\mathrm{i} \epsilon \epsilon_{k_{1}}}{\hbar}} e^{\mathrm{i} k_{1} x_{i}} \prod_{\substack{k_{2}>0 \\
\left|k_{2}\right| \neq\left|k_{1}\right|}}\left(u_{k_{2}}^{2}+v_{k_{2}}^{2} e^{-\frac{\mathrm{i} \epsilon \epsilon_{k_{2}}}{\hbar}}\right) \\
& +\sum_{k_{1}} e^{-\frac{2 \mathrm{i} \epsilon \epsilon_{1}}{\hbar}} e^{\mathrm{i} k_{1} x_{i}} v_{k_{1}}^{2} \prod_{\substack{k_{2}>0 \\
\left|k_{2}\right| \neq\left|k_{1}\right|}}\left(u_{k_{2}}^{2}+v_{k_{2}}^{2} e^{-\frac{\mathrm{i} \epsilon \epsilon_{k_{2}}}{\hbar}}\right) .
\end{aligned}
$$

\section{APPENDIX F: VORTEX SCALING}

Returning to our original initial condition of a single Bogoliubov fermion created at $x=0$, we can identify space-time vortices in the time evolved system. The discrete (exact) and CA results are compared for the TFIM in Fig. 8 where the same general trend is observed in both cases: fewer vortices at the QCP at $g=1$ than away from it at $g=0.5$. The vortices that survive at the QCP are those near to the center of the chain at $x=0$, i.e., those closest to the position of the original excitation. In fact, in the CA only a single line of vortices on each side of the center line survives.

The vortices are located by breaking the light cone up into small loops and integrating the phase of the wave function around each one. For a loop containing a single vortex,

$$
\int_{\mathcal{C}} d \chi= \pm 2 \pi
$$

where the plus sign signifies a vortex and the minus sign an antivortex. For the discrete wave function, the integral along the spatial part of the path $\mathcal{C}$ is replaced by a sum.

If we track the positions of the vortices as $g$ is varied, we find that they flow in space-time in such a way that as the QCP is approached vortices and antivortices annihilate in pairs, each pair annihilating at a different point $(x, t)$. This process is easier to follow in the CA than the discrete case because the discreteness in the lattice direction obscures the spatial location of vortices, so in this Appendix we specialize to the CA case (whereas the data presented in Fig. 6 in the main text are for the discrete case). In particular, Fig. 9 gives a pictorial representation of the annihilations occurring near the center of the lattice for $g=1.75$. We see that vortex-antivortex pairs converge on horizontal lines (i.e., spatial points) located at $x / a= \pm 0.5, \pm 1.5, \pm 2.5, \ldots$.

The temporal behavior of the vortices can also be seen in Fig. 9. For values of $g$ close the QCP, the vortex-antivortex pairs that occur at short times annihilate and so never occur, or, said another way, as $g \rightarrow 1$ the creation time for vortexantivortex pairs diverges, an example of critical slowing. Thus there are two dimensions along which one can observe critical scaling: along $t$ and along $x$, and the data for these two directions are shown in Fig. 10. It is clear from the way that the data falls onto straight lines on a log-log scale as $g \rightarrow 1$ that the vortices display critical scaling. The figure shows two "sets" of vortices, where each set annihilates within a small region of $x / a$ at diverging timescales. The vortices we call primary vortices annihilate at positions approaching $\bar{x}=1.5 a$, while $\bar{x}=2.5 a$ for the secondary vortices. In the main text, we focus only on the primary vortices, since a greater number annihilate earlier in time and thus result in a less oscillatory 


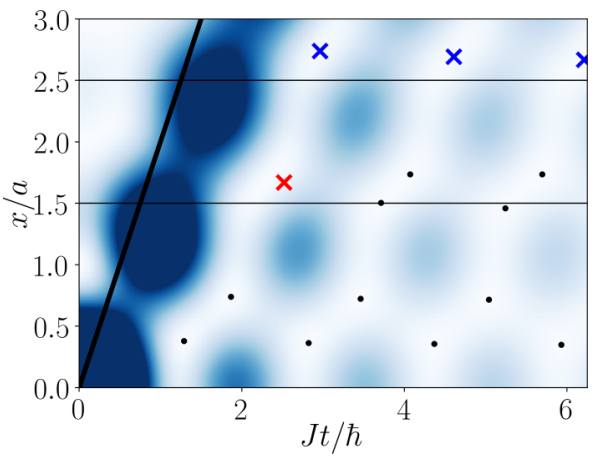

FIG. 9. Graphic depicting vortex annihilation in the CA for the TFIM. Here, $g=1.75$, and for clarity the lines $x / a=1.5$ and $x / a=2.5$ have been drawn. As $g$ is tuned toward the transition, vortex-antivortex pairs (black dots) will approach one another and eventually annihilate at a particular point in space-time, denoted with an "X." It is the vortices which annihilate close to $x / a=1.5$ that we refer to as 'primary' (red) and those which annihilate close to $x / a=2.5$ we refer to as "secondary" (blue, all annihilated in this image). In principle, there exist rows of vortices beyond these, but here we focus on those closer to the center of the lattice and short times.

integrand, allowing us to get closer to the transition while maintaining accuracy for a larger number of data points, but we see that the secondary vortices obey the same scaling. The temporal scaling shown in Fig. 10(a) leads to a gradient of -1 and hence the relation $v z=1$ as explained in the main text [see inset in Fig. 6(b)]. The spatial scaling is shown in Fig. 10(b) and leads to a gradient of 0.5 .
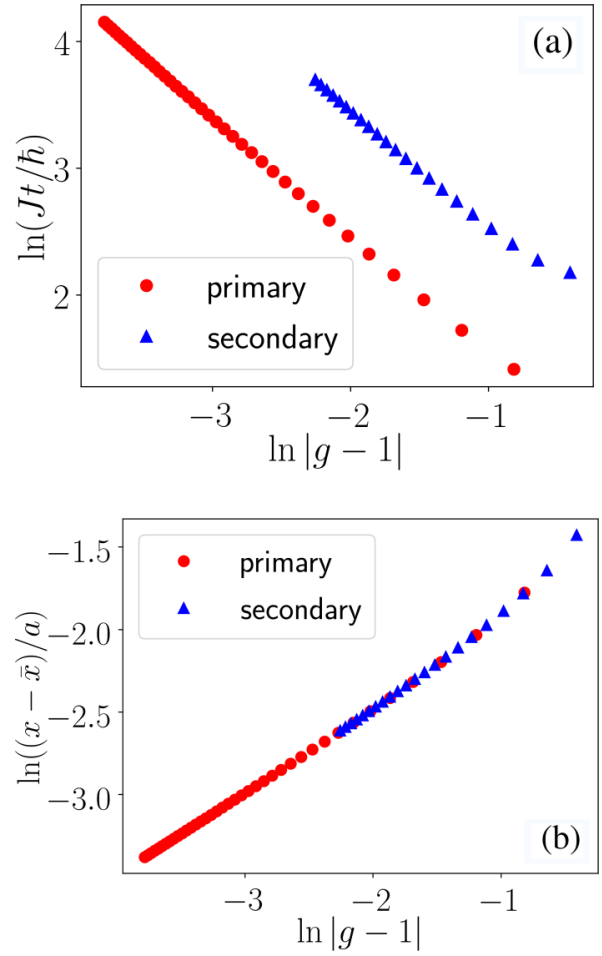

FIG. 10. Vortex annihilation scaling in the TFIM within the CA. (a) The time at which vortex annihilation occurs along a particular set of vortices will diverge as we approach the QCP. (b) Each consecutive vortex pair will annihilate at a point in space $(x)$ which approaches the midpoint between two lattice sites. Thus $\bar{x}=1.5 a$ for the set of primary vortices and $\bar{x}=2.5 a$ for the secondary vortices.
[1] E. H. Lieb and D. W. Robinson, The finite group velocity of quantum spin systems, Commun. Math. Phys. 28, 251 (1972).

[2] J. Eisert, M. Friesdorf, and C. Gogolin, Quantum many-body systems out of equilibrium, Nat. Phys. 11, 124 (2015).

[3] P. Calabrese and J. Cardy, Time Dependence of Correlation Functions Following a Quantum Quench, Phys. Rev. Lett. 96, 136801 (2006).

[4] P. Calabrese and J. Cardy, Evolution of entanglement entropy in one-dimensional systems, J. Stat. Mech. (2005) P04010.

[5] A. M. Läuchli and C. Kollath, Spreading of correlations and entanglement after a quench in the one-dimensional BoseHubbard model, J. Stat. Mech. (2008) P05018.

[6] S. R. Manmana, S. Wessel, R. M. Noack, and A. Muramatsu, Time evolution of correlations in strongly interacting fermions after a quantum quench, Phys. Rev. B 79, 155104 (2009).

[7] J.-M. Stéphan and J. Dubail, Local quantum quenches in critical one-dimensional systems: entanglement, the Loschmidt echo, and light-cone effects, J. Stat. Mech. (2011) P08019.

[8] P. Barmettler, D. Poletti, M. Cheneau, and C. Kollath, Propagation front of correlations in an interacting Bose gas, Phys. Rev. A 85, 053625 (2012).
[9] P. Calabrese, F. H. L. Essler, and M. Fagotti, Quantum quench in the transverse field Ising chain: I. Time evolution of order parameter correlators, J. Stat. Mech. (2012) P07016.

[10] J. Schachenmayer, B. P. Lanyon, C. F. Roos, and A. J. Daley, Entanglement Growth in Quench Dynamics with Variable Range Interactions, Phys. Rev. X 3, 031015 (2013).

[11] J. Eisert, M. van den Worm, S. R. Manmana, and M. Kastner, Breakdown of Quasilocality in Long-Range Quantum Lattice Models, Phys. Rev. Lett. 111, 260401 (2013).

[12] P. Hauke and L. Tagliacozzo, Spread of Correlations in LongRange Interacting Quantum Systems, Phys. Rev. Lett. 111, 207202 (2013).

[13] J. Haegeman, C. Lubich, I. Oseledets, B. Vandereycken, and F. Verstraete, Unifying time evolution and optimization with matrix product states, Phys. Rev. B 94, 165116 (2016).

[14] G. Perfetto and A. Gambassi, Ballistic front dynamics after joining two semi-infinite quantum Ising chains, Phys. Rev. E 96, 012138 (2017).

[15] M. Kormos, Inhomogeneous quenches in the transverse field Ising chain: scaling and front dynamics, SciPost Phys. 3, 020 (2017). 
[16] L. Cevolani, J. Despres, G. Carleo, L. Tagliacozzo, and L. Sanchez-Palencia, Universal scaling laws for correlation spreading in quantum systems with short- and long-range interactions, Phys. Rev. B 98, 024302 (2018).

[17] M. Cheneau, P. Barmettler, D. Poletti, M. Endres, P. Schauss, T. Fukuhara, C. Gross, I. Bloch, C. Kollath, and S. Kuhr, Light-cone-like spreading of correlations in a quantum manybody system, Nature (London) 481, 484 (2012).

[18] T. Fukuhara, P. Schauß, M. Endres, S. Hild, M. Cheneau, I. Bloch, and C. Gross, Microscopic observation of magnon bound states and their dynamics, Nature (London) 502, 76 (2013).

[19] T. Langen, R. Geiger, M. Kuhnert, B. Rauer, and J. Schmiedmayer, Local emergence of thermal correlations in an isolated quantum many-body system, Nat. Phys. 9, 640 (2013).

[20] P. Richerme, Z.-X. Gong, A. Lee, C. Senko, J. Smith, M. FossFeig, S. Michalakis, A. V. Gorshkov, and C. Monroe, Nonlocal propagation of correlations in quantum systems with long-range interactions, Nature (London) 511, 198 (2014).

[21] P. Jurcevic, B. P. Lanyon, P. Hauke, C. Hempel, P. Zoller, R. Blatt, and C. F. Roos, Quasiparticle engineering and entanglement propagation in a quantum many-body system, Nature (London) 511, 202 (2014).

[22] J. Simon, W. S. Bakr, R. Ma, M. E. Tai, P. M. Preiss, and M. Greiner, Quantum simulation of antiferromagnetic spin chains in an optical lattice, Nature (London) 472, 307 (2011).

[23] R. Islam, E. E. Edwards, K. Kim, S. Korenblit, C. Noh, H. Carmichael, G.-D. Lin, L.-M. Duan, C.-C. Joseph Wang, J. K. Freericks, and C. Monroe, Onset of a quantum phase transition with a trapped ion quantum simulator, Nat. Commun. 2, 377 (2011).

[24] K. Kim, M.-S. Chang, S. Korenblit, R. Islam, E. E. Edwards, J. K. Freericks, G.-D. Lin, L.-M. Duan, and C. Monroe, Quantum simulation of frustrated Ising spins with trapped ions, Nature (London) 465, 590 (2010).

[25] J. Struck, C. Olschlager, R. L. Targat, P. Soltan-Panahi, A. Eckardt, M. Lewenstein, P. Windpassinger, and K. Sengstock, Quantum simulation of frustrated classical magnetism in triangular optical lattices, Science 333, 996 (2011).

[26] D. Jaksch, C. Bruder, J. I. Cirac, C. W. Gardiner, and P. Zoller, Cold Bosonic Atoms in Optical Lattices, Phys. Rev. Lett. 81, 3108 (1998).

[27] M. Greiner, O. Mandel, T. Esslinger, T. W. Hänsch, and I. Bloch, Quantum phase transition from a superfluid to a Mott insulator in a gas of ultracold atoms, Nature (London) 415, 39 (2002).

[28] T. Stöferle, H. Moritz, C. Schori, M. Köhl, and T. Esslinger, Transition from a Strongly Interacting 1D Superfluid to a Mott Insulator, Phys. Rev. Lett. 92, 130403 (2004).

[29] S. Trotzky, Y.-A. Chen, A. Flesch, I. P. McCulloch, U. Schollwöck, J. Eisert, and I. Bloch, Probing the relaxation towards equilibrium in an isolated strongly correlated onedimensional Bose gas, Nat. Phys. 8, 325 (2012).

[30] T. Kinoshita, T. Wenger, and D. S. Weiss, A quantum Newton's cradle, Nature (London) 440, 900 (2006).

[31] S. Hofferberth, I. Lesanovsky, B. Fischer, T. Schumm, and J. Schmiedmayer, Non-equilibrium coherence dynamics in one-dimensional Bose gases, Nature (London) 449, 324 (2007).
[32] T. Jacqmin, J. Armijo, T. Berrada, K. V. Kheruntsyan, and I. Bouchoule, Sub-Poissonian Fluctuations in a 1D Bose Gas: From the Quantum Quasicondensate to the Strongly Interacting Regime, Phys. Rev. Lett. 106, 230405 (2011).

[33] M. Karski, L. Förster, J.-M. Choi, A. Steffen, W. Alt, D. Meschede, and A. Widera, Quantum walk in position space with single optically trapped atoms, Science 325, 174 (2009).

[34] P. M. Preiss, R. Ma, M. E. Tai, A. Lukin, M. Rispoli, P. Zupancic, Y. Lahini, R. Islam, and M. Greiner, Strongly correlated quantum walks in optical lattices, Science 347, 1229 (2015).

[35] O. Morsch and M. Oberthaler, Dynamics of Bose-Einstein condensates in optical lattices, Rev. Mod. Phys. 78, 179 (2006).

[36] R. Blatt and C. F. Roos, Quantum simulations with trapped ions, Nat. Phys. 8, 277 (2012).

[37] C. Monroe and J. Kim, Scaling the ion trap quantum processor, Science 339, 1164 (2013).

[38] W. S. Bakr, J. I. Gillen, A. Peng, S. Folling, and M. Greiner, A quantum gas microscope for detecting single atoms in a Hubbard-regime optical lattice, Nature (London) 462, 74 (2009).

[39] C. Weitenberg, M. Endres, J. F. Sherson, M. Cheneau, P. Schauß, T. Fukuhara, I. Bloch, and S. Kuhr, Single-spin addressing in an atomic Mott insulator, Nature (London) 471, 319 (2011).

[40] J. F. Sherson, C. Weitenberg, M. Endres, M. Cheneau, I. Bloch, and S. Kuhr, Single-atom-resolved fluorescence imaging of an atomic Mott insulator, Nature (London) 467, 68 (2010).

[41] J. F. Nye, Natural Focusing and Fine Structure of Light (Institute of Physics, Philadelphia, 1999).

[42] L. Kelvin, Deep water ship-waves, Philos. Mag. 9, 733 (1905).

[43] F. Ursell, Ship Hydrodynamics, Water Waves and Asymptotics. Collected works of F. Ursell, 1946-1992 (World Scientific, Singapore, 1994), Vol. 2.

[44] NIST Digital Library of Mathematical Functions, http://dlmf nist.gov/, Release 1.0.24 of 2019-09-15. F. W. J. Olver, A. B. Olde Daalhuis, D. W. Lozier, B. I. Schneider, R. F. Boisvert, C. W. Clark, B. R. Miller, B. V. Saunders, H. S. Cohl, and M. A. McClain, eds.

[45] M. V. Berry, Tsunami asymptotics, New J. Phys. 7, 129 (2005); Focused tsunami waves, Proc. R. Soc. London A 463, 3055 (2007); Minimal analytical model for undular tidal bore profile; quantum and Hawking effect analogies, New J. Phys. 20, 053066 (2018).

[46] V. L. Ginzburg, Radiation from uniformly moving sources (Vavilov-Cherenkov effect, transition radiation, and some other phenomena), Acoust. Phys. 51, 11 (2005).

[47] I. Carusotto, S. X. Hu, L. A. Collins, and A. Smerzi, Bogoliubov-Čerenkov Radiation in a Bose-Einstein Condensate Flowing against an Obstacle, Phys. Rev. Lett. 97, 260403 (2006).

[48] Y. G. Gladush, L. A. Smirnov, and A. M. Kamchatnov, Generation of Cherenkov waves in the flow of a Bose-Einstein condensate past an obstacle, J. Phys. B 41, 165301 (2008).

[49] J. Marino, A. Recati, and I. Carusotto, Casimir Forces and Quantum Friction from Ginzburg Radiation in Atomic Bose-Einstein Condensates, Phys. Rev. Lett. 118, 045301 (2017). 
[50] R. Thom, Structural Stability and Morphogenesis (Benjamin, Reading MA, 1975).

[51] V. I. Arnol'd, Critical points of smooth functions and their normal forms, Russ. Math. Survs. 30, 1 (1975).

[52] M. Berry, Singularities in Waves and Rays in Les Houches, Session XXXV, 1980 Physics of Defects, edited by R. Balian et al. (North Holland, Amsterdam, 1981).

[53] E. Lieb, T. Schultz, and D. Mattis, Two soluble models of an antiferromagnetic chain, Ann. Phys. 16, 407 (1961).

[54] S. Katsura, Statistical mechanics of the anisotropic linear heisenberg model, Phys. Rev. 127, 1508 (1962).

[55] P. G. de Gennes, Collective motions of hydrogen bonds, Solid State Commun. 1, 132 (1963).

[56] P. Pfeuty, The one-dimensional Ising model with a transverse field, Ann. Phys. (NY) 57, 79 (1970).

[57] J. Häppölä, G. B. Halász, and A. Hamma, Universality and robustness of revivals in the transverse field XY model, Phys. Rev. A 85, 032114 (2012).

[58] T. Langen, T. Schweigler, E. Demler, and J. Schmiedmayer, Double light-cone dynamics establish thermal states in integrable 1D Bose gases, New J. Phys. 20, 023034 (2018).

[59] V. Eisler and Z. Rácz, Full Counting Statistics in a Propagating Quantum Front and Random Matrix Spectra, Phys. Rev. Lett. 110, 060602 (2013).

[60] J. Viti, J.-M. Stéphan, J. Dubail, and M. Haque, Inhomogeneous quenches in a free fermionic chain: Exact results, Europhys. Lett. 115, 40011 (2016).

[61] V. Eisler and F. Maislinger, Hydrodynamical phase transition for domain-wall melting in the XY chain, Phys. Rev. B. 98, 161117(R) (2018).

[62] V. Hunyadi, Z. Rácz, and L. Sasvári, Dynamic scaling of fronts in the quantum XX chain, Phys. Rev. E 69, 066103 (2004).

[63] K. Najafi, M. A. Rajabpour, and J. Viti, Light-cone velocities after a global quench in a noninteracting model, Phys. Rev. B. 97, 205103 (2018).

[64] N. Allegra, J. Dubail, J.-M. Stéphan, and J. Viti, Inhomogeneous field theory inside the arctic circle, J. Stat. Mech. (2016) 053108 .

[65] S. Xu and B. Swingle, Accessing scrambling using matrix product operators, arXiv:1802.00801.

[66] C.-J. Lin and O. I. Motrunich, Out-of-time-ordered correlators in quantum Ising chain, Phys. Rev. B 97, 144304 (2018).

[67] J. Riddell and E. S. Sørensen, Out-of-time ordered correlators and entanglement growth in the random-field XX spin chain, Phys. Rev. B 99, 054205 (2019).

[68] J. Riddell and E. S. Sørensen, Out of time order correlations in the quasi-periodic aubry-andré model, arXiv:1908.03292

[69] T. C. Petersen, M. Weyland, D. M. Paganin, T. P. Simula, S. A. Eastwood, and M. J. Morgan, Electron Vortex Production and Control Using Aberration Induced Diffraction Catastrophes, Phys. Rev. Lett. 110, 033901 (2013).

[70] W. Rooijakkers, S. Wu, P. Striehl, M. Vengalattore, and M. Prentiss, Observation of caustics in the trajectories of cold atoms in a linear magnetic potential, Phys. Rev. A 68, 063412 (2003).

[71] J. H. Huckans, I. B. Spielman, B. L. Tolra, W. D. Phillips, and J. V. Porto, Quantum and classical dynamics of a BoseEinstein condensate in a large-period optical lattice, Phys. Rev. A 80, 043609 (2009).
[72] S. Rosenblum, O. Bechler, I. Shomroni, R. Kaner, T. ArusiParpar, O. Raz, and B. Dayan, Demonstration of Fold and Cusp Catastrophes in an Atomic Cloud Reflected from an Optical Barrier in the Presence of Gravity, Phys. Rev. Lett. 112, 120403 (2014).

[73] J. T. Chalker and B. Shapiro, Caustic formation in expanding condensates of cold atoms, Phys. Rev. A 80, 013603 (2009).

[74] T. P. Simula, T. C. Petersen, and D. M. Paganin, Diffraction catastrophes threaded by quantized vortex skeletons caused by atom-optical aberrations induced in trapped Bose-Einstein condensates, Phys. Rev. A 88, 043626 (2013).

[75] U. Leonhardt, A laboratory analog of the event horizon using slow light in an atomic medium, Nature (London) 415, 406 (2002).

[76] M. V. Berry and M. R. Dennis, Quantum cores of optical phase singularities, J. Opt. A 6, S178 (2004).

[77] M. V. Berry, Three quantum obsessions, Nonlinearity 21, T19 (2008).

[78] D. H. J. O'Dell, Quantum Catastrophes and Ergodicity in the Dynamics of Bosonic Josephson Junctions, Phys. Rev. Lett. 109, 150406 (2012).

[79] J. Mumford, W. Kirkby, and D. H. J. O'Dell, Catastrophes in non-equilibrium many-particle wave functions: universality and critical scaling, J. Phys. B 50, 044005 (2017).

[80] J. Mumford, E. Turner, D. W. L. Sprung, and D. H. J. O’Dell, Quantum Spin Dynamics in Fock Space Following Quenches: Caustics and Vortices, Phys. Rev. Lett. 122, 170402 (2019).

[81] M. V. Berry, Attenuation and focusing of electromagnetic surface waves rounding gentle bends, J. Phys. A 8, 566 (1975).

[82] S. Sachdev, Quantum Phase Transitions, 2nd ed. (Cambridge University Press, New York, 2011).

[83] P. Calabrese, F. H. L. Essler, and M. Fagotti, Quantum Quench in the Transverse-Field Ising Chain, Phys. Rev. Lett. 106, 227203 (2011).

[84] L. Bucciantini, Light-cone effect and relaxation after a quantum quench from excited states in the Ising chain, J. Phys.: Conf. Ser. 566, 012026 (2014).

[85] M. Karl, H. Cakir, J. C. Halimeh, M. K. Oberthaler, M. Kastner, and T. Gasenzer, Universal equilibrium scaling functions at short times after a quench, Phys. Rev. E 96, 022110 (2017).

[86] M. V. Berry and C. Upstill, Catastrophe optics: morphologies of caustics and their diffraction patterns, Prog. Opt. 18, 257 (1980).

[87] E. Nicklas, M. Karl, M. Höfer, A. Johnson, W. Muessel, H. Strobel, J. Tomkovič, T. Gasenzer, and M. K. Oberthaler, Observation of Scaling in the Dynamics of a Strongly Quenched Quantum Gas, Phys. Rev. Lett. 115, 245301 (2015).

[88] M. V. Berry, J. F. Nye, and F. J. Wright, The elliptic umbilic diffraction catastrophe, Philos. Trans. R. Soc. Lond. 291, 1382 (1979).

[89] J. F. Nye and M. V. Berry, Dislocations in wave trains, Proc. R. Soc. Lond. A 336, 165 (1974).

[90] D. Kaminski and R. B. Paris, On the zeros of the Pearcey integral, J. Comput. Appl. Math. 107, 31 (1999).

[91] J. F. Nye, Dislocation lines in the swallowtail diffraction catastrophe, Proc. R. Soc. A 462, 2299 (2006).

[92] J. E. Bunder and R. H. McKenzie, Effect of disorder on quantum phase transitions in anisotropic $\mathrm{XY}$ spin chains in a transverse field, Phys. Rev. B 60, 344 (1999). 
[93] A. Dutta, G. Aeppli, B. Chakrabarti, U. Divakaran, T. Rosenbaum, and D. Sen, Quantum Phase Transitions in Transverse Field Spin Models: From Statistical Physics to Quantum Information (Cambridge University Press, Cambridge, 2015).

[94] G. Lemarié, H. Lignier, D. Delande, P. Szriftgiser, and J.-C. Garreau, Critical State of the Anderson Transition: Between a Metal and an Insulator, Phys. Rev. Lett. 105, 090601 (2010).

[95] J. D. Ring, J. Lindberg, A. Mourka, M. Mazilu, K. Dholakia, and M. R. Dennis, Auto-focusing and self-healing of Pearcey beams, Opt. Express 20, 18955 (2012).

[96] T. Poston and I. Stewart, Catastrophe Theory And Its Applications (Dover, Minola, New York, 1996).

[97] R. Gilmore, Catastrophe Theory for Scientists and Engineers (Wiley, New York, 1981).

[98] R. Gilmore, Structural stability of the phase transition in dickelike models, J. Math. Phys. 18, 17 (1977).

[99] R. Gilmore and L. M. Narducci, Relation between the equilibrium and nonequilibrium critical properties of the dicke model, Phys. Rev. A 17, 1747 (1978).
[100] J. T. Stone, R. H. Self, and C. J. Howls, Aeroacoustic catastrophes: Upstream cusp beaming in Lilley's equation, Proc. R. Soc. A 473, 20160880 (2017).

[101] V. I. Arnold, S. F. Shandarin, and Ya. B. Zeldovich, The large scale structure of the universe I. general properties. one- and two-dimensional models, Geophys. Astrophys. Fluid Dyn. 20, 111 (1982).

[102] J. Feldbrugge, R. van de Weygaert, J. Hidding, and J. Feldbrugge, Caustic skeleton \& cosmic web, J. Cosmol. Astropart. Phys. 2018, 27 (2018).

[103] V. I. Arnold, Mathematical Methods of Classical Mechanics (Springer, New York, 1997)

[104] B. Gardas, J. Dziarmaga, and W. H. Zurek, Dynamics of the quantum phase transition in the one-dimensional BoseHubbard model: Excitations and correlations induced by a quench, Phys. Rev. B 95, 104306 (2017).

[105] A. D. Zaikin, D. S. Golubev, A. van Otterlo, and G. T. Zimanyi, Quantum fluctuations and dissipation in thin superconducting wires, Usp. Fiz. Nauk 168, 244 (1998) [Phys. Usp. 41, 226 (1998)]. 